\title{
ON THE EXTENSION OF DELAUNAY'S METHOD IN THE LUNAR THEORY TO THE GENERAL PROBLEM OF PLANETARY MOTION*
}

\author{
BY
}

\author{
G. W. HILL
}

\section{Part I.-Exposition of the Theory.}

The method of integrating the differential equations of motion, adopted by Delaunay for the elaboration of his lunar theory, as it is explained by him, demands its division into several cases, and is established through very tedious transformations. These disadvantages disappear when the greatest generality is given to the procedure. Hence, an explanation of the method, as it would be applied to the motion of a planetary system like the solar, will, doubtless, be welcome to astronomers.

I.

Let $T$ denote the living force of the system, $\Omega$ the potential function, and, with PoIncare, put

$$
F=\Omega-T .
$$

The $k$ variables, necessary for completely defining the position of the system, may be denoted by $q_{1}, q_{2}, \cdots, q_{k}$. Use accents to denote complete differentiation with respect to the time of the latter, and we have

$$
T=\text { function }\left(q_{1}, q_{2}, \cdots, q_{k}, q_{1}^{\prime}, q_{2}^{\prime}, \cdots, q_{k}^{\prime}\right) .
$$

The partial derivatives of this function with respect to the $k$ variables $q_{i}^{\prime}$ are to be used as variables instead of the latter, and we put

$$
p_{i}=\frac{\partial T}{\partial q_{i}^{\prime}}, \quad(i=1,2, \cdots, k)
$$

By means of these $k$ equations the $q_{i}^{\prime}$ can be eliminated from $T$, and thus will result :

$$
T=\text { function }\left(q_{1}, q_{2}, \cdots, q_{k}, p_{1}, p_{2}, \cdots, p_{k}\right) .
$$

\footnotetext{
* Presented to the Society February 24, 1900. Received for publication February 5, 1900.
} 
Then the system of differential equations for determining the variables $p_{i}$ and $q_{i}$ is :

$$
\frac{d p_{i}}{d t}=\frac{\partial F}{\partial q_{i}}, \quad \frac{d q_{i}}{d t}=-\frac{\partial F}{\partial p_{i}}, \quad(i=1,2, \cdots, k) .
$$

Let us suppose that $\Omega$ is separated into the two parts $\Omega_{0}$ and $\Omega_{1}$, and that, when we neglect $\Omega_{1}$ in $F$, the equations (1) can be completely integrated, and their integrals expressed in terms of two sets of $k$ quantities each, symbolized thus :

$$
\begin{gathered}
L_{1}, L_{2}, \cdots, L_{k}, \\
\lambda_{1}, \lambda_{2}, \cdots, \lambda_{k},
\end{gathered}
$$

of which the first set are constants, and the second set linear functions of the time of the form $n_{i} t+c_{i}, n_{i}$ being a function of the $L_{i}$, and $c_{i}$ an arbitrary constant. Nothing forbids our taking the $L_{i}$ such that they may be the elements severally conjugate to the $\lambda_{i}$.

Now, desiring to integrate the equations (1) when $F$ has its complete value, we may adopt the $L_{i}$ and the $\lambda_{i}$ as the dependent variables to be employed. The differential equations of the problem are then :

$$
\frac{d L_{i}}{d t}=\frac{\partial F}{\partial \lambda_{i}}, \quad \frac{d \lambda_{i}}{d t}=-\frac{\partial F}{\partial L_{i}}, \quad(i=1,2, \cdots, k)
$$

Here the function $F$ has been made to involve the $L_{i}$ and $\lambda_{i}$ by eliminating the old variables $p_{i}$ and $q_{i}$ from it by means of their values given by the integrals derived on the supposition that $\Omega=\Omega_{0}$. As

$$
F=\text { a constant }
$$

is an integral of the problem, and $\Omega_{0}-T=$ a constant, when $\Omega_{1}$ is neglected, it is quite evident that when we substitute in $\Omega_{0}-T$ for $p_{i}$ and $q_{i}$ their values in terms of the $L_{i}$ and $\lambda_{i}$, the $\lambda_{i}$ completely disappear and $\Omega_{0}-T$ becomes a function of the $L_{i}$ only. Thus, in the second form for $F$, the variables $\lambda_{i}$ enter into it solely through the portion $\Omega_{1}$.

\section{II.}

In order to exemplify we will adduce the solar system composed of the Sun and the eight major planets. We will suppose that the masses of the Sun, Mercury, Venus, $\cdots$, Neptune are denoted by $m_{0}, m_{1}, m_{2}, \cdots, m_{8}$, and will put

$$
\mu_{i}=m_{0}+m_{1}+m_{2}+\cdots+m_{i}, \quad \kappa_{i}=\frac{m_{i}}{\mu_{i}}, \quad(i=0,1, \cdots, 8) .
$$


Let the type of representation of the rectangular coördinates of the planets relative to the Sun be as follows:

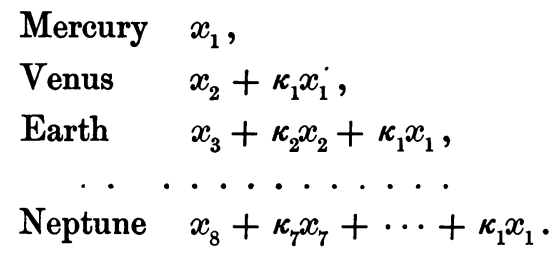

The differential equations these variables satisfy are :

$$
\left\{\begin{array}{l}
\mu_{i-1} \kappa_{i} \frac{d^{2} x_{i}}{d t^{2}}=\frac{\partial \Omega}{\partial x_{i}}, \\
\mu_{i-1} \kappa_{i} \frac{d^{2} y_{i}}{d t^{2}}=\frac{\partial \Omega}{\partial y_{i}}, \\
\mu_{i-1} \kappa_{i} \frac{d^{2} z_{i}}{d t^{2}}=\frac{\partial \Omega}{\partial z_{i}},
\end{array} \quad(i=1,2, \cdots, 8)\right.
$$

Here $\Omega$ denotes the sum of the products of every two masses of the system divided by their distance, a relation we will write thus :

$$
\Omega=m_{0} \sum \frac{m_{i}}{\Delta_{0, i}}+\sum \frac{m_{i} m_{j}}{\Delta_{i, j}} .
$$

Suppose that the portion to be separated from $\Omega$ is

$$
\Omega_{0}=m_{0} \sum \frac{m_{i}}{r_{i}}
$$

$r_{i}$ standing for $\sqrt{\left(x_{i}^{2}+y_{i}^{2}+z_{i}^{2}\right)}$. Then, if $\Omega_{0}$ is substituted for $\Omega$ in equations (3), and the members are divided by $\mu_{i-1} \kappa_{i}$, we get

$$
\left\{\begin{array}{l}
\frac{d^{2} x_{i}}{d t^{2}}+m_{0} \frac{\mu_{i}}{\mu_{i-1}} \frac{x_{i}}{r_{i}^{3}}=0 \\
\frac{d^{2} y_{i}}{d t^{2}}+m_{0} \frac{\mu_{i}}{\mu_{i-1}} \frac{y_{i}}{r_{i}^{3}}=0, \\
\frac{d^{2} z_{i}}{d t^{2}}+m_{0} \frac{\mu_{i}}{\mu_{i-1}} \frac{z_{i}}{r_{i}^{3}}=0,
\end{array} \quad(i=1,2, \cdots, 8)\right.
$$

It will be seen that each group of these equations, corresponding to the same value of $i$, is independent of all the rest, and that it differs from the group of equations of relative motion of two bodies only in that the constant $m_{0} \mu_{i} / \mu_{i-1}$ takes the place of $m_{0}+m_{i}$. 
Let $a_{i}$ be the semi-axis major, $e_{i}$ the eccentricity, $\phi_{i}$ the inclination, $l_{i}$ the mean anomaly, $g_{i}$ the angular distance of the perihelion from the node, and $h_{i}$ the longitude of the node. Put

$$
\left\{\begin{array}{l}
L_{i}=\sqrt{m_{0} \frac{\mu_{i}}{\mu_{i-1}} a_{i}}, \\
G_{i}=L_{i} \sqrt{1-e_{i}^{2}}, \\
H_{i}=G_{i} \cos \phi_{i}
\end{array} \quad(i=1,2, \cdots, 8)\right.
$$

Then, when the elements become variable by reason of the addition of $\Omega_{1}$ to $\Omega_{0}$, they will satisfy the differential equations :

$$
\left\{\begin{array}{ll}
\frac{d L_{i}}{d t}=\frac{\partial R_{i}}{\partial l_{i}}, & \frac{d l_{i}}{d t}=-\frac{\partial R_{i}}{\partial L_{i}}, \\
\frac{d G_{i}}{d t}=\frac{\partial R_{i}}{\partial g_{i}}, & \frac{d g_{i}}{d t}=-\frac{\partial R_{i}}{\partial G_{i}}, \\
\frac{d H_{i}}{d t}=\frac{\partial R_{i}}{\partial h_{i}}, & \frac{d l_{i}}{d t}=-\frac{\partial R_{i}}{\partial H_{i}},
\end{array} \quad(i=1,2, \cdots, 8),\right.
$$

where $R_{i}$ will be, in terms of $\Omega_{\mu}$, mentioned above,

$$
R_{i}=\frac{m_{0} \frac{\mu_{i}}{\mu_{i-1}}}{2 a_{i}}+\frac{\Omega_{j}}{\mu_{i-1} \kappa_{i}}, \quad(i=1,2, \cdots, 8) .
$$

Desiring to have the same perturbative function, whatever may be the integer $i$, we multiply the values (7) of $L_{i}, G_{i}, H_{i}$, as also the value (9) of $R_{i}$ by the constant $\mu_{i-1} \kappa_{i}$, which does not alter the form of equation ${ }_{\Lambda}(8)$. We now have :

as also :

$$
\left\{\begin{array}{l}
L_{i}=m_{i} \sqrt{m_{0} \frac{\mu_{i-1}}{\mu_{i}} a_{i}}, \\
G_{i}=L_{i} \sqrt{1-e_{i}^{2}}, \\
H_{i}=G_{i} \cos \phi_{i},
\end{array} \quad(i=1,2, \cdots, 8),\right.
$$

$$
F=m_{0} \sum_{i=1}^{i=8} \frac{m_{i}}{2 a_{i}}+m_{0} \sum_{i=2}^{i=\infty} m_{i}\left[\frac{1}{\Delta_{0, i}}-\frac{1}{r_{i}}\right]+\sum \frac{m_{i} m_{j}}{\Delta_{i, j}}
$$

If the planetary coördinates in the last equation are replaced by the elements $(10)$ and the $l_{i}, g_{i}, h_{i}$, the differential equations of the system are : 


$$
\left\{\begin{array}{rll}
\frac{d L_{i}}{d t}=\frac{\partial F}{\partial l_{i}} ; & \frac{d l_{i}}{d t}=-\frac{\partial F}{\partial L_{i}}, & \\
\frac{d G_{i}}{d t}=\frac{\partial F}{\partial g_{i}}, & \frac{d g_{i}}{d t}=-\frac{\partial F}{\partial G_{i}}, & (i=1,2, \cdots, 8) . \\
\frac{d H_{i}}{d t}=\frac{\partial F}{\partial h_{i}}, & \frac{d h_{i}}{d t}=-\frac{\partial F}{\partial H_{i}},
\end{array}\right.
$$

In the second term of the right member of (11) the quantities $1 / \Delta_{0,2}, 1 / \Delta_{0,3}$, $\ldots, 1 / \Delta_{0,8}$, can be developed in infinite series, the first terms of which are $1 / r_{2}, 1 / r_{3}, \cdots, 1 / r_{8}$, and thus are cancelled by the term $1 / r_{i}$. Then the two latter terms of (11) are of the second order with reference to planetary masses.

III.

In order to make the application of Delaunay's method it appears necessary that $F$ should be developed in a series, finite or infinite and periodic with respect to the variables $l_{i}, g_{i}, h_{i}$, which have been named the angular variables. In astronomical problems the series are generally infinite. For legitimate employment this series must remain convergent throughout the whole duration of motion, while $t$ is passing from $-\infty$ to $+\infty$. It becomes then pertinent to ask what conditions must be fulfilled in order that this series may be convergent. It is well known that the reciprocal of the distance between two planets can be developed in a convergent infinite series, periodic with respect to the mean anomalies of the planets, provided that the orbits, as they stand in space, have no point in common, or when the reciprocal of the distance never becomes infinite. The condition of convergence in the present case is precisely similar to this. Here, however, not only the mean anomalies $l_{i}$ are left indeterminate in the series, but also the remaining angular variables $g_{i}$ and $h_{i}$ which define the positions of the perihelia and nodes. Hence, in the present case, there must not only be no actual intersection of the orbits, but none when the perihelia and nodes are shifted in every possible way, the linear variables, or the mean distances, eccentricities and inclinations retaining their actual values. In the Delaunay development of the reciprocal of the distance between two planets, it is necessary and it suffices for convergence that the perihelion radius of one of the planets should always exceed the aphelion radius of the other.

We may consider this subject under a more general aspect. Let $F$ have the periodic development

$$
F=\sum A \cos \left[j_{1} \lambda_{1}+j_{2} \lambda_{2}+\cdots+j_{k} \lambda_{k}\right],
$$

where $\lambda_{1} \cdots \lambda_{k}$ are the angular variables, the $j$ positive or negative integers, and $A$ is a function of the linear variables $L$ only. That this infinite series may be 
convergent, $F$ must not only actually never become infinite, but never even potentially so. It is necessary here to explain what we mean by the qualifying epithet " potentially." If, while the linear variables $L$ are supposed to maintain their actual values, and, consequently, the coefficients $A$ their actual values, we allow to all the angular variables $\lambda$ the complete swing of movement from 0 to $2 \pi$, $\boldsymbol{F}$ remains always finite, we say it never potentially becomes infinite. In order that $F$ may not actually become infinite it is necessary and sufficient that the velocities of and the distances between the points of the system should remain finite. In order that $F$ may not potentially become infinite, it is necessary and sufficient that the values of the linear variables $L_{1}, L_{2}, \ldots, L_{k}$ should remain within a certain domain. The definition of this domain is very complex after a Delaunay transformation has been operated, but is quite simple in terms of the original Keplerian linear variables $L_{1}, L_{2}, \ldots, L_{k}$.

We may illustrate this subject by bringing forward the case of the solar system as it has been described in $\S$ II. Employing the linear elements $a_{i}$ and $e_{i}$ of equation (10) or of equations (7), the inequalities which define this domain are :

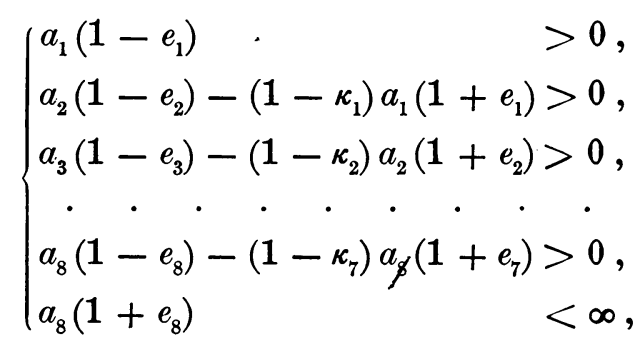

or, if we prefer to use the elements $L_{i}$ and $G_{i}$ of the inequalities are :

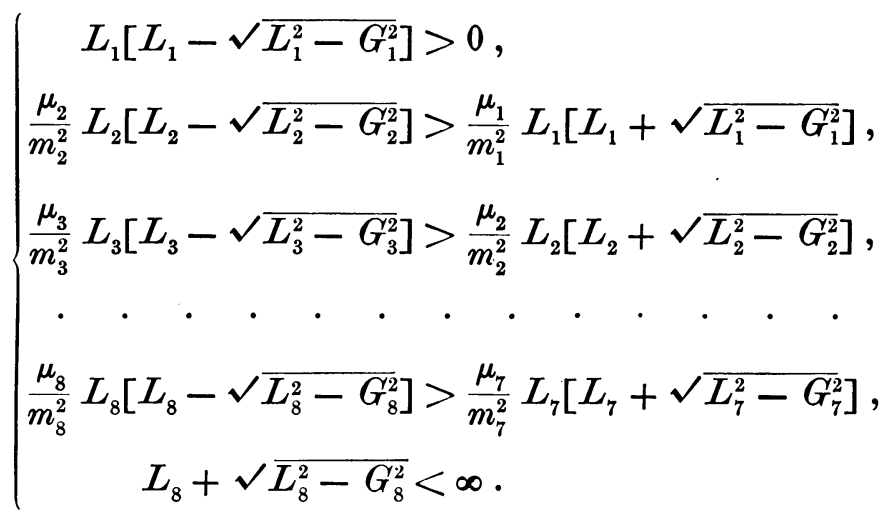

It is remarkable that the inclinations of the orbits play no part in these inequalities. 
IV.

As, in general, it is not necessary to distinguish between the three kinds $l_{i}, g_{i}, h_{i}$ of angular variables, nor between the three kinds $L_{i}, G_{i}, H_{i}$, of linear variables, for simplicity of notation we shall suppose that the angular variables are denoted by $l_{1}, l_{2}, \ldots, l_{k}$, and their corresponding conjugate linear variables by $L_{1}, L_{2}, \cdots, L_{k}$.

Selecting a particular linear combination $\theta$ of of the angular variables so that

$$
\theta=j_{1} l_{1}+j_{2} l_{2}+\cdots+j_{k} l_{k}
$$

the $j$ being positive or negative integers prime to each other, Delaunay's method, somewhat generalized, consists in making such a transformation of variables as would constitute a complete solution of the problem if $F$, in its periodic development, contained as arguments only integral multiples of $\theta$. That is, in this special case, the new linear variables would turn out constants, and the new angular variables would be of the form $n(t+c), n$ and $c$ being likewise constants. It is clear that when we make such a transformation in $F$, the terms in the former periodic development involving the cosines of the finite multiples of $\theta$ will disappear, but the absolute term will receive a modification. A little consideration will make it evident that the derivation of such a transformation is dependent on quadratures.

The discussion of this derivation is greatly facilitated by making a linear transformation of variables, in the cases of both angular and linear variables. In this it is evident that we can take $\theta$ as one of the angular variables; then let $\Theta$ be its conjugate linear variable. Thus, we may have the following as the variables involved in the problem :

$$
\begin{gathered}
\text { Linear variables, } \Theta, \Lambda_{1}, \Lambda_{2}, \ldots, \Lambda_{k-1} ; \\
\text { Angular variables, } \theta, \lambda_{1}, \lambda_{2}, \ldots, \lambda_{k-1} .
\end{gathered}
$$

And the canonical system of differential equations will be :

$$
\left\{\begin{array}{ccc}
\frac{d \Theta}{d t}=\frac{\partial F}{\partial \theta}, & \frac{d \theta}{d t}=-\frac{\partial F}{\partial \Theta}, \\
\frac{d \Lambda_{1}}{d t}=\frac{\partial F}{\partial \lambda_{1}}, & \frac{d \lambda_{1}}{d t}=-\frac{\partial F}{\partial \Lambda_{1}}, \\
\cdot \quad \cdot \quad \cdot & \cdot \quad \cdot \quad \cdot \\
\frac{d \Lambda_{k-1}}{d t}=\frac{\partial F}{\partial \lambda_{k-1}}, & \frac{d \lambda_{k-1}}{d t}=-\frac{\partial F}{\partial \Lambda_{k-1}} .
\end{array}\right.
$$

Let us now consider the mean value of the function $F$ relatively to the angular 
variables $\lambda_{1}, \lambda_{2}, \ldots \lambda_{k-1}$. Then, since $F$ as a periodic function involves only cosines of arguments, if $[F]$ denote the mentioned mean value, we shall have

$$
[F]=\left[\frac{1}{\pi} \int_{0}^{\pi}\right]^{k-1} F d \lambda_{1} d \lambda_{2} \cdots d \lambda_{k-1},
$$

where the first factor of the right member denotes an operation repeated $k-1$ times, once in reference to each of the variables $\lambda_{1}, \lambda_{2}, \ldots, \lambda_{k-1}$. As $F$ remains finite whatever values $\theta, \lambda_{1}, \lambda_{2}, \cdots, \lambda_{k-1}$ may assume, it follows that $[F]$ is finite whatever may be the value of $\theta$. Thus $[F]$ is developable as a periodic function of $\theta$ involving only cosines; and we may write

$$
[F]=-B-A_{1} \cos \theta-A_{2} \cos 2 \theta-A_{3} \cos 3 \theta-\cdots,
$$

where $B, A_{1}, A_{2}, A_{3}, \ldots$ are functions of the linear variables $\Theta, \Lambda_{1}, \Lambda_{2}$, $\cdots, \Lambda_{k-1}$.

Let us now suppose that, in equations $(16),[F]$ is substituted for $F$. They then become :

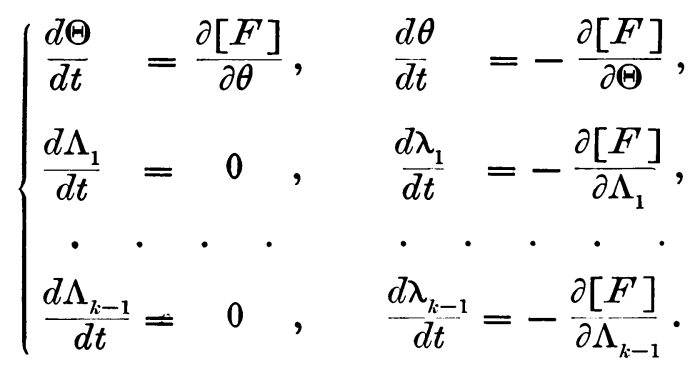

$\Lambda_{1}, \Lambda_{2}, \ldots, \Lambda_{k-1}$ are therefore constants, and the two equations of the first line contain no other variables than $\Theta$ and $\theta$, and thus form a distinct system by themselves and determine these two variables; after which, by substitution of values, the remaining differential equations for $\lambda_{1}, \lambda_{2}, \ldots, \lambda_{k-1}$ determine these variables through quadratures.

V.

As $[F]$ involves only two variables $\Theta$ and $\theta$, the two equations which begin (19) have the integral, $C$ being an arbitrary constant,

$$
[F]+C=0 \text {. }
$$

This integral constitutes a relation between the two variables $\Theta$ and $\theta$; and, if the latter are regarded as coördinates defining the position of a point in a plane, (20) is the equation of a plane curve. For this graphical exhibition of the connection between the two variables, we might adopt that in which they are 
the polar coördinates of a point, $\Theta$ being the radius and $\theta$ the angle. But, in some cases $\Theta$ may pass through zero. This difficulty may be obviated by adding to it a sufficiently large positive constant and thus it be rendered uniformly positive. This can be done provided it does not go to negative infinity. However, all circumstances considered, it will probably be a better course to adopt a representation in rectangular coördinates, $\theta$ being the abscissa and $\Theta$ the ordinate.

If we derive from (20) an expression for $\theta$ in terms of $\Theta$ and substitute it in the first equation of (19) and take the reciprocal of both members we shall have the time in terms of $\Theta$ by a quadrature, and, by the inversion of this, $\Theta$ as a function of $t$. On the other hand, if we derive from (20) an expression for $\Theta$ in terms of $\theta$, and substitute it in the second equation of $(19)$ we shall have the time in terms of $\theta$ by a quadrature, and, by the inversion of this, $\theta$ as a function of $t$.

We proceed to note some of the properties of the curve whose equation is (20). In the first place it must be stated that if the differential equations of (19), which determine the variables $\Theta$ and $\theta$, compel the first of these to take on values rendering the right member of (18) a divergent series, we agree to set aside such cases as nugatory. Singularities of a certain kind are therefore excluded. The curve cannot have a point $d$ 'arrêt, for, at this point, we should have simultaneously $d \Theta / d t=0$ and $d \theta / d t=0$; and in consequence all succeeding derivatives of these variables would vanish. Thus, at this point $\Theta$ and $\theta$ would be invariable, which is impossible. It cannot have a multiple point, since, for given values of $\Theta$ and $\theta$, there is but one value of each of the quantities $d \Theta / d t$ and $d \theta / d t$. If the curve pass through a point, it must proceed thence until it returns to that point or goes on to infinity. In the latter case, taking a polar representation for the moment, it may either have two infinite branches, or may make an infinite number of turns about the pole, or, in other words be a spiral. But, since equation (20) involves only cosines of $\theta$ without sines of the same, the curve must needs be symmetrically situated with respect to the axis from which $\theta$ is measured. Hence, the last supposition must be rejected ; that is, it cannot be a spiral, nor can it have more than one distinct turn about the pole.

The curves graphically representing (20) may be divided into three classes. Here, for convenience, we adopt a rectangular representation. Let us suppose that an infinite number of values between the limits 0 and $\pi$ are substituted for $\theta$ in $(20)$; the result will be an infinite number of equations for determining the corresponding values of $\Theta$. Let one of these be satisfied by a real value of $\Theta$. Then it may happen that all the remaining equations are satisfied by real values of this variable continuous among themselves and with the value first mentioned. The variable $\theta$ can then move from $-\infty$ to $+\infty$ and there will always be a corresponding real value for $\Theta$. The first equation of (19) shows that $\Theta$ will be at 
a maximum or minimum when $\theta=i \pi, i$ being a positive or negative integer. As, in the equation

$$
\frac{d \Theta}{d t}=A_{1} \sin \theta+2 A_{2} \sin 2 \theta+3 A_{3} \sin 3 \theta+\cdots,
$$

the quantity $A_{1}$ is, in general, larger than $A_{2}, A_{3}, \ldots$, it follows that $\Theta$ will have no other maximum or minimum values than those just mentioned. In addition, if a maximum value occurs for $\theta=2 i \pi$, then will a minimum value occur for $\theta=(2 i+1) \pi$, and vice versa. If, in (20) we put, in succession $\theta=0, \theta=\pi$, we shall have the two equations :

$$
\left\{\begin{array}{l}
C-B=A_{1}+A_{2}+A_{3}+\cdots \\
C-B=-A_{1}+A_{2}-A_{3}+\cdots
\end{array}\right.
$$

And if $\Theta$ be regarded as the unknown to be determined by them, it is plain that the maximum value of $\Theta$ will be a root of one of them and the minimum value a root of the other. Again $\Theta$ cannot be constant unless all the coefficients $A$ vanish. It is quite evident that, in this case, the values of $\Theta$ and $\theta$ can be represented by the infinite periodic series :

$$
\left\{\begin{array}{l}
\Theta=\Theta_{0}+\Theta_{1} \cos \left[\theta_{0}(t+c)\right]+\Theta_{2} \cos 2\left[\theta_{0}(t+c)\right]+\cdots \\
\theta=\theta_{0}(t+c)+\theta_{1} \sin \left[\theta_{0}(t+c)\right]+\theta_{2} \sin 2\left[\theta_{0}(t+c)\right]+\cdots
\end{array}\right.
$$

These two equations are to be regarded as the integrals of the first and second differential equations of the group (19); $c$ is one of the arbitrary constants introduced by the integration, the other may be supposed to be either the $C$ of (20) or the $\Theta_{0}$ of the first of (23). But while $C$ and $c$ are conjugate to each other, this is not necessarily the case with the elements $\Theta_{0}$ and $\theta_{0}(t+c)$. The remaining coefficients of $(23), \Theta_{1}, \Theta_{2}, \ldots, \theta_{0}, \theta_{1}, \theta_{2}, \ldots$, are functions of $C$ or $\Theta_{0}$. On account of the form of the curve which represents (20) in this case it may be called the sinusoid case.

We come now to consider the second case of the representation of (20) by a curve. Here, if we give to $\theta$ its range of values between 0 and $\pi$, we shall find that the equations determining the corresponding values of $\Theta$ have two real roots for an arc of values for $\theta$ which either begins at 0 or ends at $\pi$; and, in the first case the arc terminates, or, in the second case, begins, at the same intermediate point. At this point the two real roots become equal, and, for the remainder of the semi-circumference, they are imaginary. Consequently, at this point, $\theta$ attains either a maximum or minimum value. Because the equation contains only cosines of multiples of $\theta$, in the one case, the right line $\theta=0$, and, in the other, the right line $\theta=\pi$, divides the area embraced by the curve symmetrically. The maximum and minimum values of $\Theta$ are given by the roots of that 
one of the two equations of (22) which has two real roots. In this case, $\theta$ can not be represented by series like the second of (23), but, in general, we may give the integrals of the problem the form :

$$
\left\{\begin{array}{l}
\Theta \cos \theta=P_{0}+P_{1} \cos \left[\theta_{0}(t+c)\right]+P_{2} \cos 2\left[\theta_{0}(t+c)\right]+\cdots, \\
\Theta \sin \theta=\quad Q_{1} \sin \left[\theta_{0}(t+c)\right]+Q_{2} \sin 2\left[\theta_{0}(t+c)\right]+\cdots,
\end{array}\right.
$$

where $\theta_{0}, P_{0}, P_{1}, P_{2}, \cdots, Q_{1}, Q_{2}, \cdots$, are constant coefficients and functions of the $C$ of $(20)$, while $c$, as before, is the other arbitrary constant. It will be perceived that, in the former case, the integral equations (23) can be given the form (24) if one chooses; and Delaunay has always adopted it where the eccentricity $e$ would appear as a divisor in the first form. At the two points, at which $\theta$ has attained its maximum or minimum value, we have $d \theta / d t=0$, or

$$
\frac{d B}{d \Theta}+\frac{d A_{1}}{d \Theta} \cos \theta+\frac{d A_{2}}{d \Theta} \cos 2 \theta+\cdots=0 .
$$

When $d B / d \Theta$ and $d A_{1} / d \Theta$ are quantities of the same order of magnitude, the second case is likely to occur. As the curve, which here represents the connection of the variables $\Theta$ and $\theta$, is a closed one, this case may be called the ovaloid case. This kind of motion in the variables is, however, generally termed a libration. Observation has not yet shown that it occurs in the system of the eight major planets of the solar system, although it is possible it may exist for very large values of the integers $j_{i}$. However, should this prove true, the influence of this circumstance on the motion of the system would be quite insignificant.

The third case in the graphical representation of (20) occurs when, in a certain range of values for $\theta$, bisected by the value $\theta=0$ or by the value $\theta=\pi$, we find a real value for $\Theta$, but this value tends towards positive or negative infinity as the limits are approached. Here there is one maximum and no minimum for $\Theta$ or one minimum and no maximum. As in the previous cases, these values occur when $\theta=0$ or $\theta=\pi$. As long as the instantaneous orbits of the planets composing the system are elliptic in their nature this case cannot present itself. And $\Theta$ cannot go beyond a certain limit without some of the elements becoming imaginary. In order, therefore, to prevent the occurrence of functions of complex variables, a modified system has to be adopted. But an illustration of this case can very easily be constructed. In order to escape the difficulty of divergence when $|\Theta|$ exceeds a certain limit, let us suppose that $[F]$ is finite and does not run into an infinite series, and that all the quantities $A_{i}$ beyond $A_{1}$ vanish. Then the equation (20), being solved with reference to $\cos \theta$, gives

$$
\cos \theta=\frac{C-B}{A_{1}}=\frac{f(\Theta)}{F(\Theta)}
$$


Let $\Theta_{0}$ be the value of $\Theta$ when $\theta=0$; in order to have the present case we ought to have

$$
\frac{f\left(\Theta_{0}\right)}{F\left(\Theta_{0}\right)}=1, \quad \frac{f(\infty)}{F(\infty)}=a
$$

$a$ being less than unity. We may suppose that $\Theta$ is involved linearly in $B$ and $A_{1}$, so that, $a, b, c, d$ being constants,

Then

$$
f(\Theta)=a+b \Theta, \quad F(\Theta)=c+d \Theta .
$$

$$
\Theta_{0}=\frac{a-c}{d-b}, \quad a=\frac{b}{d} .
$$

All the conditions will be fulfilled if we put

$$
f(\Theta)=3+4 \Theta, \quad F(\Theta)=2+5 \Theta ;
$$

whence $\Theta_{0}=1$ and $a=\frac{4}{5}$. $\Theta$ is thus continuous while $\theta$ is contained between the two values given by the equation $\cos \theta=\frac{4}{5}$. At the limits $\Theta$ becomes infinite. In a system of polar coördinates, if $\Theta$ is the radius and $\theta$ the angle, the equation of the curve graphically exhibiting the connection of the variables $\Theta$ and $\theta$ is :

$$
\cos \theta=\frac{3+4 \Theta}{2+5 \Theta}, \text { or } \Theta=\frac{-3+2 \cos \theta}{4-5 \cos \theta} \text {. }
$$

It is thus a quartic algebraic curve whose equation in rectangular coördinates is :

$$
\left[2 x-4\left(x^{2}+y^{2}\right)\right]^{2}=(3-5 x)^{2}\left(x^{2}+y^{2}\right),
$$

whose course resembles that of a hyperbola. The formula for the time is :

$$
t+c=\int \frac{d \Theta}{\sqrt{(2+5 \Theta)^{2}-(3+4 \Theta)^{2}}}=\frac{1}{3} \int \frac{d \Theta}{\sqrt{\left(\Theta-\frac{2}{9}\right)^{2}-\left(\frac{7}{9}\right)^{2}}} .
$$

If this be integrated between the limits $\Theta=1$ and $\Theta=\Theta$ it will give the time required to describe the curve from the point $\theta=0$ to the point having the radius $\Theta$.

VI.

Let us now suppose that by the integration of the system of differential equations (19), it is proposed to remove from $F$ the periodic terms having the argument $\theta$, that is, those contained in $[F]$. We confine ourselves to the first case as that will usually be the one which presents itself. The integrals of (19) will evidently have the form : 


$$
\begin{aligned}
& \left\{\begin{array}{l}
\Theta=\Theta_{0}+\Theta_{1} \cos \left[\theta_{0}(t+c)\right]+\Theta_{2} \cos 2\left[\theta_{0}(t+c)\right]+\cdots, \\
\Lambda_{1}=\text { a constant }, \quad \Lambda_{2}=\text { a constant }, \cdots, \quad \Lambda_{k-1}=\text { a constant }
\end{array}\right. \\
& \theta=\theta_{0}(t+c)+\theta_{1} \sin \left[\theta_{0}(t+c)\right]+\theta_{2} \sin 2\left[\theta_{0}(t+c)\right]+\cdots, \\
& \left\{\lambda_{1}=\left(\lambda_{1}\right)+\lambda_{1}^{(0)}(t+c)+\lambda_{1}^{(1)} \sin \left[\theta_{0}(t+c)\right]+\lambda_{1}^{(2)} \sin 2\left[\theta_{0}(t+c)\right]+\cdots,\right. \\
& \lambda_{2}=\left(\lambda_{2}\right)+\lambda_{2}^{(0)}(t+c)+\lambda_{2}^{(1)} \sin \left[\theta_{0}(t+c)\right]+\lambda_{2}^{(2)} \sin 2\left[\theta_{0}(t+c)\right]+\cdots, \\
& \lambda_{k-1}=\left(\lambda_{k-1}\right)+\lambda_{k-1}^{(0)}(t+c)+\lambda_{k-1}^{(1)} \sin \left[\theta_{0}(t+c)\right]+\lambda_{k-1}^{(2)} \sin 2\left[\theta_{0}(t+c)\right]+\cdots,
\end{aligned}
$$

where $c,\left(\lambda_{1}\right),\left(\lambda_{2}\right), \cdots,\left(\lambda_{k-1}\right), \Lambda_{1}, \Lambda_{2}, \cdots, \Lambda_{k-1}$, and one other involved in $\Theta_{0}, \Theta_{1}, \ldots, \theta_{0}, \lambda_{1}^{(0)}, \lambda_{1}^{(1)}, \ldots$, complete the number of $2 k$ arbitrary constants. If then we take formulas of transformation as follows, replacing

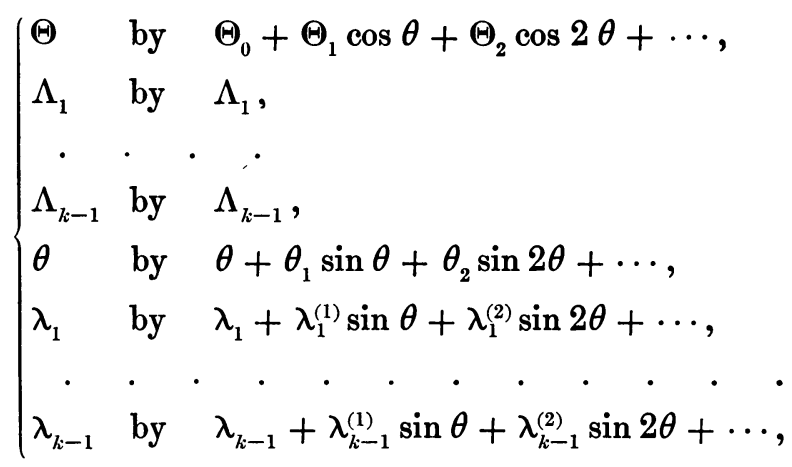

the terms of $F$ involving $\theta$ as the argument will disappear from it, their mean values going over into the non-periodic term $-B$.

We shall now have a new set of angular variables $\theta, \lambda_{1}, \lambda_{2}, \ldots, \lambda_{k-1}$, and it will be pertinent to inquire what are the linear variables conjugate to them, so that we may still have differential equations of the canonical form. Taking the two equations of (27) which define $\Theta$ and $\theta$ in terms of the new variables, let us suppose that the latter are $\Theta_{0}$ and $\theta^{\prime}$ (an accent is applied to the latter for the sake of discrimination). Then we have

$$
\left\{\begin{array}{c}
\Theta=\Theta_{0}+\Theta_{1} \cos \theta^{\prime}+\Theta_{2} \cos 2 \theta^{\prime}+\cdots, \\
\theta=\theta^{\prime}+\theta_{1} \sin \theta^{\prime}+\theta_{2} \sin 2 \theta^{\prime}+\cdots .
\end{array}\right.
$$

Then, by the substitution of these values in the two differential equations of (16). which determine $\Theta$ and $\theta$, we get

$$
\left\{\begin{array}{l}
\frac{\partial \Theta}{\partial \Theta_{0}} \frac{d \Theta_{0}}{d t}+\frac{\partial \Theta}{\partial \theta^{\prime}} \frac{d \theta^{\prime}}{d t}=\frac{\partial F^{\prime}}{\partial \Theta_{0}} \frac{\partial \Theta_{0}}{\partial \theta}+\frac{\partial F}{\partial \theta^{\prime}} \frac{\partial \theta^{\prime}}{\partial \theta} \\
\frac{\partial \theta}{\partial \Theta_{0}} \frac{d \Theta_{0}}{d t}+\frac{\partial \theta}{\partial \theta^{\prime}} \frac{d \theta^{\prime}}{d t}=-\frac{\partial F}{\partial \Theta_{0}} \frac{\partial \Theta_{0}}{\partial \Theta}-\frac{\partial F}{\partial \theta^{\prime}} \frac{\partial \theta^{\prime}}{\partial \Theta} .
\end{array}\right.
$$


By multiplying these equations by the proper factors, and putting $\Delta$ for the functional determinant or Jacobian :

we have

$$
\Delta=\frac{\partial \Theta}{\partial \Theta_{0}} \frac{\partial \theta}{\partial \theta^{\prime}}-\frac{\partial \Theta}{\partial \theta^{\prime}} \frac{\partial \theta}{\partial \Theta_{0}},
$$

But

$$
\Delta \frac{d \Theta_{0}}{d t}=\frac{\partial F}{\partial \theta^{\prime}}, \quad \Delta \frac{d \theta^{\prime}}{d t}=-\frac{\partial F}{\partial \Theta_{0}} .
$$

$$
\begin{array}{r}
\Delta=\left[1+\frac{\partial \Theta_{1}}{\partial \Theta_{0}} \cos \theta^{\prime}+\frac{\partial \Theta_{2}}{\partial \Theta_{0}} \cos 2 \theta^{\prime}+\cdots\right]\left[1+\theta_{1} \cos \theta^{\prime}+2 \theta_{2} \cos 2 \theta^{\prime}+\cdots\right] \\
+\left[\Theta_{1} \sin \theta^{\prime}+2 \Theta_{2} \sin 2 \theta^{\prime}+\cdots\right] \cdot\left[\frac{\partial \theta_{1}}{\partial \Theta_{0}} \sin \theta^{\prime}+\frac{\partial \theta_{2}}{\partial \Theta_{0}} \sin 2 \theta^{\prime}+\cdots\right]
\end{array}
$$

According to the theorem of Poisson, $\Delta$ is independent of $t$, or what in this case amounts to the same thing, of $\theta^{\prime}$. Hence, in computing its value, we have regard only to the absolute term. Thus

$$
\begin{aligned}
\Delta & =1+\frac{1}{2}\left[\theta_{1} \frac{\partial \Theta_{1}}{\partial \Theta_{0}}+2 \theta_{2} \frac{\partial \Theta_{2}}{\partial \Theta_{0}}+\cdots+\Theta_{1} \frac{\partial \theta_{1}}{\partial \Theta_{0}}+2 \Theta_{2} \frac{\partial \theta_{2}}{\partial \Theta_{0}}+\cdots\right] \\
& =1+\frac{1}{2} \frac{\partial}{\partial \Theta_{0}}\left[\theta_{1} \Theta_{1}+2 \theta_{2} \Theta_{2}+3 \theta_{3} \Theta_{3}+\cdots\right] .
\end{aligned}
$$

Then, if we adopt a new variable $\Theta^{\prime}$ in place of $\Theta_{0}$ such that

$$
\Theta^{\prime}=\int \Delta d \Theta_{0}=\Theta_{0}+\frac{1}{2}\left[\theta_{1} \Theta_{1}+2 \theta_{2} \Theta_{2}+3 \theta_{3} \Theta_{3}+\cdots\right],
$$

equations (31) will be transformed into :

$$
\frac{d \Theta^{\prime}}{d t}=\frac{\partial F^{\prime}}{\partial \theta^{\prime}}, \quad \frac{d \theta^{\prime}}{d t}=-\frac{\partial F^{\prime}}{\partial \Theta^{\prime}},
$$

which have the canonical form. As to the remaining linear variables $\Lambda_{1}, \Lambda_{2}$, $\ldots, \Lambda_{k-1}$, which are identical with the former variables denoted by the same symbols, it is evident that they remain the conjugates of the new variables $\lambda_{1}$, $\lambda_{2}, \ldots, \lambda_{k-1}$.

VII.

As it is somewhat difficult to discover the linear transformation of variables required to pass from the set

$$
\left.\left.\begin{array}{c}
L_{1}, L_{2}, \ldots, L_{k} \\
l_{1}, l_{2}, \ldots, l_{k}
\end{array}\right\} \text { to the set } \begin{array}{r}
\Theta, \Lambda_{1}, \Lambda_{2}, \ldots, \Lambda_{k-1} \\
\theta, \lambda_{1}, \lambda_{2}, \ldots, \lambda_{k-1}
\end{array}\right\},
$$


suppose we adhere to the use of the first set. Then we have the differential equations

$$
\begin{aligned}
& \frac{d \Theta}{d \bar{t}}=\frac{\partial[F]}{\partial \theta}, \quad \frac{d \theta}{d t}=-\frac{\partial[F]}{\partial \Theta}, \\
& \frac{d L_{i}}{d t}=j_{i} \frac{\partial[F]}{\partial \theta}, \quad \frac{d l_{i}}{d t}=-\frac{\partial[F]}{\partial L_{i}}, \quad(i=1,2, \cdots, k) .
\end{aligned}
$$

Then

$$
L_{i}=\left(L_{i}\right)+j_{i} \Theta, \quad(i=1,2, \cdots, k),
$$

where the $\left(L_{i}\right)$ are arbitrary constants. By means of these equations the linear variables $L_{i}$ may be eliminated from the right members of all the differential equations (36) and (37), and thus be replaced by the single variable $\Theta$. Thus the mentioned right members become functions only of the two variables $\Theta$ and $\theta$; and, after $\Theta$ and $\theta$ are found in terms of $t$ from the integration of (36), the $L_{i}$ will be found from (38), and the $l_{i}$ from a quadrature operated on the second equations of (37).

Then we can suppose that the integrals of (36) and (37) are written thus :

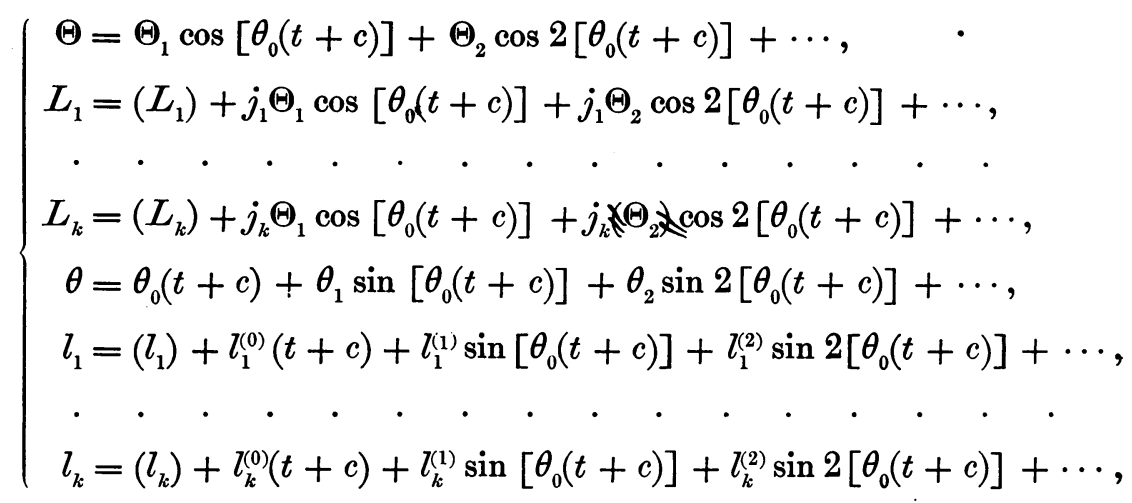

where the $\left(L_{1}\right),\left(L_{2}\right), \ldots,\left(L_{k}\right),\left(l_{1}\right),\left(l_{2}\right), \ldots,\left(l_{k}\right)$ are arbitrary constants. The latter set are not independent as they must satisfy the relation :

$$
j_{1}\left(l_{1}\right)+j_{2}\left(l_{2}\right)+\cdots+j_{k}\left(l_{k}\right)=0 .
$$

As there is the additional arbitrary constant $c$, the number of independent constants is $2 k$ as it should be. The coefficients $\theta_{0}, l_{i}^{(0)}, l_{i}^{(1)}, l_{i}^{(2)}, \ldots$, which may be regarded as functions of the $\left(L_{i}\right)$, satisfy the relations :

$$
\begin{aligned}
& j_{1}^{l_{1}^{(0)}}+j_{2} l_{2}^{(0)}+\cdots+j_{k} l_{k}^{(0)}=\theta_{0}, \\
& j_{1} l_{1}^{(1)}+j_{2} l_{2}^{(1)}+\cdots+j_{k} l_{k}^{(1)}=\theta_{1},
\end{aligned}
$$


The formulas of transformation are then :

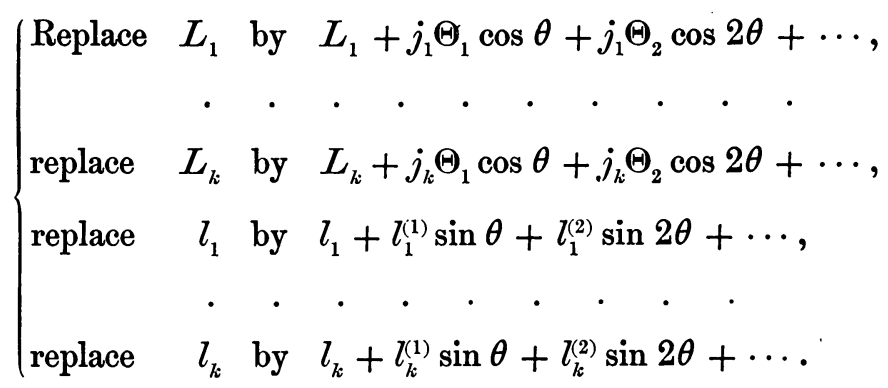

We have now to find what linear variables are conjugate to the new angular variables $l_{1}, l_{2}, \cdots l_{k}$. They are discovered immediately from a comparison of (34) and (38). As, from (34), it appears that $\Theta_{0}$ the arbitrary constant, which may be conceived as annexed to the series for $\Theta$, is not the element conjugate to the angular element $\theta_{0}(t+c)$, but that the expression

$$
\frac{1}{2}\left[\theta_{1} \Theta_{1}+2 \theta_{2} \Theta_{2}+3 \theta_{3} \Theta_{3}+\cdots\right]
$$

must be added to it to produce the required conjugate, it is plain from (38) that, after the transformation, the new $L_{i}$ is no longer the exact conjugate of $l_{i}$, but that we have for that element the value

$$
L_{i}+\frac{1}{2} j_{i}\left[\theta_{1} \Theta_{1}+2 \theta_{2} \Theta_{2}+3 \theta_{3} \Theta_{3}+\cdots\right] \quad(i=1,2, \cdots, k) .
$$

\section{VIII.}

In making one of DeLAunay's transformations it is not absolutely necessary that we should employ the linear variables $L_{i}$, which are the conjugates of the angular variables $l_{i}$, in the development of the various series needed; we may use any others connected with the former by known relations. Then equation (41) will inform us, at any stage of the transformations, what function the conjugates of the angular variables are of the used linear variables. Thus, in making his developments in the lunar theory, Delaunay has not used the elements he calls $L, G, H$, but has substituted for them others which he names $a, e, \gamma$.

Let us suppose that the new set of linear elements we determine to use are denoted by the symbols $e_{1}, e_{2}, \cdots, e_{k}$. Then, in order to form the equivalents for the $d l_{i} / d t$, it will be necessary to know the values of the partial derivatives $\partial e_{i} / \partial L_{j}$ in terms of the $e_{i}$. The number of these derivatives is $k^{2}$, and we shall have as many equations for determining them. Having the $L_{i}$ in terms of the $e_{i}$, the general form of these equations will be :

$$
\frac{\partial L_{i}}{\partial e_{1}} \frac{\partial e_{1}}{\partial L_{j}}+\frac{\partial L_{i}}{\partial e_{2}} \frac{\partial e_{2}}{\partial L_{j}}+\cdots+\frac{\partial L_{i}}{\partial e_{k}} \frac{\partial e_{k}}{\partial L_{j}}=0 \text { or } 1
$$


according as $i$ and $j$ are different or are the same. These equations divide themselves into $k$ groups each containing $k$ equations; each group serving to determine the $k$ partial derivatives of the $e_{i}$ with reference to one of the $L_{i}$ which we denote by $L_{j}$. The functional determinant of each group of equations is the same, being the Jacobian of the variables $L_{1}, L_{2}, \ldots, L_{k}$ with reference to the variables $e_{1}, e_{2}, \cdots, e_{k}$. Calling this determinant $\Delta$, we shall have

$$
\frac{\partial e_{i}}{\partial L_{j}}=\frac{1}{\Delta} \frac{\partial \Delta}{\partial\left(\frac{\partial L_{j}}{\partial e_{i}}\right)} .
$$

We shall much abbreviate the calculation of these partial derivatives by agreeing to compute $\partial e_{i} / \partial L_{j}$ not directly but by a correction to be added to the value it had before the transformation was made. This correction will be denoted by $D\left(\partial e_{i} / \partial L_{j}\right)$. Now the transformation being that made to remove from $F$ all the periodic terms having the argument

$$
j_{1} l_{1}+j_{2} l_{2}+\cdots+j_{k} l_{k}
$$

suppose that, in this transformation, we put

and also

$$
K=\frac{1}{2}\left[\theta_{1} \Theta_{1}+2 \theta_{2} \Theta_{2}+3 \theta_{3} \Theta_{3}+\cdots\right],
$$

$$
-K_{m}=\frac{\partial K}{\partial e_{1}}\left(\frac{\partial e_{1}}{\partial L_{m}}\right)+\frac{\partial K}{\partial e_{2}}\left(\frac{\partial e_{2}}{\partial L_{m}}\right)+\cdots+\frac{\partial K}{\partial e_{k}}\left(\frac{\partial e_{k}}{\partial L_{m}}\right)
$$

where $\left(\partial e_{i} / \partial L_{m}\right)$ denotes the value of the partial derivative before the transformation; then we shall have the group of linear equations :

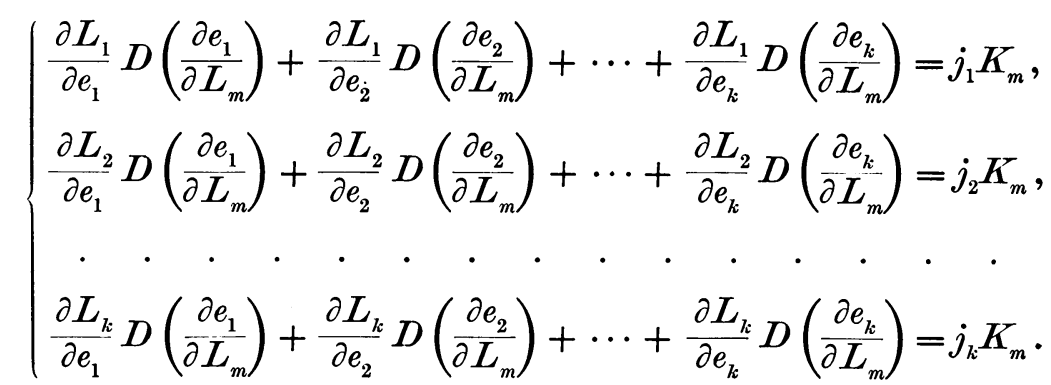

Put now

Then, evidently,

$$
P_{i}=j_{1} \frac{\partial \Delta}{\partial\left(\frac{\partial L_{1}}{\partial e_{i}}\right)}+j_{2} \frac{\partial \Delta}{\partial\left(\frac{\partial L_{2}}{\partial e_{i}}\right)}+\cdots+j_{k} \frac{\partial \Delta}{\partial\left(\frac{\partial L_{k}}{\partial e_{i}}\right)} .
$$

$$
D\left(\frac{\partial e_{i}}{\partial L_{m}}\right)=\frac{P_{i} K_{m}}{\Delta} .
$$


Thus the corrections for each partial derivative, caused by a given transformation, are reduced to the product of three factors, one of which is a function of $i$, another a function of $m$, and the third independent of either. The elements $e_{i}$, before the performance of any transformation, may be what we choose. Delaunay chose his $a, e, \gamma$ so that they were connected with $L, G, H$, the conjugates of the angular variables $l, g, h$, by the relations :

$$
L=\sqrt{\mu a}, \quad G=L \sqrt{1-e^{2}}, \quad H=G\left(1-2 \gamma^{2}\right) .
$$

\section{PART II.}

\section{Application of Delaunay's Method to the Minor Planet of the Hecuba Type.}

IX.

Delaunay's lunar theory affords a plentiful assortment of the transformations just discussed, but their application in a case of planetary motion gives rise to more complex expressions. In the lunar theory it is possible to expand all coefficients in power series of all the parameters involved; but, in a planetary theory where $a$, the ratio of the mean distances, is a considerable fraction, it is necessary to introduce the functions of $a$ usually denoted by the symbol $b_{s}^{(i)}$, as also their derivatives with respect to $a$. It may therefore be profitable to give as simple an illustration as possible of these transformations where $b_{s}^{(i)}$ must be used.

Let Jupiter be supposed to describe a circular orbit about the Sun, while a small planet, without mass, describes an orbit in the same plane. Let the radius of Jupiter's orbit be taken as the linear unit ; denote its longitude by $\epsilon^{\prime}+n^{\prime} t$, and the masses severally of the Sun and Jupiter by $m_{0}$ and $m^{\prime}$. Let $a, e, l$, and $g$ be the mean distance, eccentricity, mean anomaly, and longitude of the perihelion of the small planet, and $r$ and $v$ its radius and true anomaly. Put

$$
\gamma=g-\epsilon^{\prime}-n^{\prime} t, \quad L=\sqrt{m_{0} a}, \quad \Gamma=\sqrt{m_{0} a\left(1-e^{2}\right)} .
$$

The function we have denoted by $F$ will have the expression :

$$
F^{\prime}=\frac{m_{0}}{2 a}+n^{\prime} \Gamma+m^{\prime}\left\{\left[1-2 r \cos (v+\gamma)+r^{2}\right]^{-\frac{1}{2}}-r \cos (v+\gamma)\right\}
$$

where $r$ and $v$ are to be eliminated through the equations :

$$
r \cos v=a(\cos u-e), \quad r \sin v=a \sqrt{1-e^{2}} \sin u, \quad u-e \sin u=l .
$$

The position of the small planet will be known when we know $L, \Gamma, l, \gamma$. 
The differential equations for determining the latter are :

$$
\begin{cases}\frac{d L}{d t}=\frac{\partial F}{\partial l}, & \frac{d l}{d t}=-\frac{\partial F}{\partial L} \\ \frac{d \Gamma}{d t}=\frac{\partial F}{\partial \gamma}, & \frac{d \gamma}{d t}=-\frac{\partial F}{\partial \Gamma} .\end{cases}
$$

$\mathbf{X}$.

In order to give an illustration of the transformations named by DELAUNAY operations, let us select from the periodic development of $F$, which, from (49), plainly has the form $\sum A_{i, i^{\prime}} \cos \left(i l+i^{\prime} \gamma\right)$, all the terms having the argument $\theta=l+2 \gamma$. These will be terms of long period in case the small planet has a mean motion nearly double that of Jupiter, which case has been extensively dis. cussed by astronomers, such a minor planet being called of the Hecuba type. Taking $\theta$ as one of the angular elements, we see that we can adopt $\gamma$ as the other, and thus shall have $l=\theta-2 \gamma$. In order to obtain $[F]$ from $F$ we have the equation :

$$
\left[F^{\prime}\right]=\frac{m_{0}}{2 a}+n^{\prime} \Gamma+\frac{1}{\pi} \int_{0}^{\pi} \frac{m^{\prime} d \gamma}{\sqrt{1-2 r \cos (v+\gamma)+r^{2}}},
$$

remembering that $r$ and $v$ are now the same functions of $\theta-2 \gamma$ they were before of $l$. The last term of $F$ in (49) is here omitted as it contributes nothing to $[F]$. Put

$$
\begin{aligned}
{\left[1-2 r \cos (v+\gamma)+r^{2}\right]^{-1}=\frac{1}{2} B^{(0)}+} & B^{(1)} \cos (v+\gamma) \\
& +B^{(2)} \cos 2(v+\gamma)+\cdots,
\end{aligned}
$$

where $B^{(i)}$ is the same function of $r$ that $b_{\frac{1}{2}}^{(i)}$ is of $a$. In order that this series may be convergent it is necessary that $a(1+e)<1$. Let us put

Then we have

$$
A^{(i)}=\frac{1}{\pi} \int_{0}^{\pi} B^{(2 i)} \cos i(2 v-l) d l .
$$

$$
\begin{aligned}
{[F]=\frac{m_{0}}{2 a}+n^{\prime} \sqrt{m_{0} a\left(1-e^{2}\right)}+m^{\prime}\left[\frac{1}{2} A^{(0)}+\right.} & A^{(1)} \cos \theta \\
& \left.+A^{(2)} \cos 2 \theta+\cdots\right] .
\end{aligned}
$$

The investigation will be facilitated if we now make a slight change in the dependent variables employed so that they have the following equivalents :

$$
\begin{cases}\Theta=\sqrt{m_{0} a}, & \Gamma=\sqrt{m_{0} a}\left[2-\sqrt{1-e^{2}}\right], \\ \theta=l+2 g-2 \epsilon^{\prime}-2 n^{\prime} t, & \gamma=\epsilon^{\prime}+n^{\prime} t-g .\end{cases}
$$


Then the differential equations determining the formulas of transformation are :

$$
\begin{cases}\frac{d \Theta}{d t}=\frac{\partial[F]}{\partial \theta}, & \frac{d \theta}{d t}=-\frac{\partial[F]}{\partial \Theta}, \\ \frac{d \Gamma}{d t}=0, & \frac{d \gamma}{d t}=-\frac{\partial[F]}{\partial \Gamma} .\end{cases}
$$

Of these equations the integral of the third, $\Gamma=$ a constant, furnishes the relation :

$$
a=a\left[2-\sqrt{1-e^{2}}\right]^{-2}=a\left[1-e^{2}+\frac{1}{2} e^{4}-\frac{1}{4} e^{6}+\frac{3}{32} e^{8}-\cdots\right],
$$

$a$ being a constant. By means of this relation the variable $a$ may be eliminated from $[F]$ which thus will contain but two variables, $e$ and $\theta$. The equations (56) have the canonical form, but we prefer to discard the variable $\Gamma$ and to use $e$ in its stead. Supposing then that $[F]$ is made a function of the variables $e$ and $\theta$, the differential equations for the latter are :

$$
\left\{\begin{array}{l}
\frac{d e}{d t}=-\frac{1}{\sqrt{m_{0} a}} \frac{\sqrt{1-e^{2}}\left[2-\sqrt{1-e^{2}}\right]^{2}}{e} \frac{\partial[F]}{\partial \theta}, \\
\frac{d \theta}{d t}=\frac{1}{\sqrt{m_{0} a}} \frac{\sqrt{1-e^{2}}\left[2-\sqrt{1-e^{2}}\right]^{2}}{e} \frac{\partial[F]}{\partial e},
\end{array}\right.
$$

where the factor

$$
\frac{\sqrt{1-e^{2}}\left[2-\sqrt{1-e^{2}}\right]^{2}}{e}=\frac{1}{e}\left[1+\frac{1}{2} e^{2}-\frac{1}{8} e^{4}-\frac{3}{16} e^{6}-\frac{17}{128} e^{8}-\cdots\right] \text {. }
$$

These equations form a group to be integrated by themselves. After this integration is accomplished, $\gamma$ is derived through a quadrature of the equation :

$$
\frac{d \gamma}{d t}=-\frac{\partial[F]}{\partial \Gamma}
$$

In this equation $[F]$ is a function of $\Theta$ and $\Gamma$, but we have preferred to write it as a function of $a$ and $e$; thus :

$$
\frac{\partial[F]}{\partial \Gamma}=\frac{\partial[F]}{\partial a} \frac{\partial a}{\partial \mathbf{I}^{\bar{\gamma}}}+\frac{\partial[F]}{\partial e} \frac{\partial e}{\partial \Gamma} .
$$

But

$$
\begin{aligned}
\Theta & =\frac{\sqrt{m_{0} a}}{2-\sqrt{1-e^{2}}}, & \Gamma & =\sqrt{m_{0} a}, \\
a & =\frac{\Gamma^{2}}{m_{0}}, & \sqrt{1-e^{2}} & =2-\frac{\Gamma}{\Theta} .
\end{aligned}
$$


Consequently

$$
\frac{\partial a}{\partial \Gamma}=2 \sqrt{\frac{a}{m_{0}}}, \quad \frac{\partial e}{\partial \Gamma}=\frac{1}{\sqrt{m_{0} a}} \frac{\sqrt{1-e^{2}}\left[2-\sqrt{1-e^{2}}\right]}{e} .
$$

Remembering that, with our adopted linear unit, $a^{\prime}=1, n^{\prime}=\sqrt{m_{0}+m^{\prime}}$, we have :

$$
\overline{1} \bar{n}^{\prime} \frac{d \gamma}{d t}=-\frac{1}{m_{0} \sqrt{\left(1+\frac{m^{\prime}}{m_{0}}\right) a}}\left[2 a \frac{\partial[F]}{\partial a}+\frac{\sqrt{1-e^{2}}\left[2-\sqrt{1-e^{2}}\right]}{e} \frac{\partial[F]}{\partial e}\right] .
$$

But, adopting, as before, $g$ for the longitude of the peribelion, this is more simply :

$$
\begin{aligned}
\frac{1}{n^{\prime}} \frac{d g}{d t}= & \frac{1}{\sqrt{\left(1+\frac{m^{\prime}}{m_{0}}\right) \phi \phi^{2}}}\left[2 a \frac{\partial R}{\partial a}+\frac{\sqrt{1-e^{2}}\left[2-\sqrt{\left.1-e^{2}\right]}\right.}{e} \frac{\partial R}{\partial e}\right] \\
= & \frac{2}{\sqrt{\left(1+\frac{m^{\prime}}{m_{0}}\right) a}} a \frac{\partial R}{\partial a}+\frac{1}{2-\sqrt{1-e^{2}}} \frac{1}{n^{\prime}} \frac{d \theta}{d t} \\
& -\frac{1}{\sqrt{\left(1+\frac{m^{\prime}}{m_{0}}\right) a}}\left[2-\sqrt{1-e^{2}}\right]^{2}+\frac{2}{2-\sqrt{1-e^{2}}},
\end{aligned}
$$

where

$$
R=\frac{m^{\prime}}{m_{0}} \frac{1}{\pi} \int_{0}^{\pi} \frac{d \gamma}{\sqrt{1-2 r \cos (v-\gamma)+r^{2}}}
$$

XI.

In an application like the present, where the periodic developments of the various quantities are always tardily convergent, it is nearly impossible to give literal expressions for the coefficients. And, even if we consent to give to each coefficient its numerical value at once, the work of multiplying such periodic series together is very embarrassing, and the process easily leads to the commission of errors. Hence we adopt the method of substituting for each quantity involved the special values of it at equal intervals in the motion of the independent variable through the semicircumference. With this method of treatment it is necessary to separate the cases of non-libration and libration.

It is always an advantage in computation to have the members dealt with in dependent of any linear and temporal units. To this end let us substitute for 
the independent variable $t$, the variable $\tau=\epsilon^{\prime}+n^{\prime} t$ or the longitude of Jupiter; also we put

$$
W=\frac{[F]}{m_{0}}, \quad \nu=\frac{m^{\prime}}{m_{0}}
$$

The coefficients of the periodic development of $W$ are then absolute numbers. The equations which, with (57), we shall use for the elaboration of the problem, are the three following equations :

$$
\left\{\begin{array}{l}
\text { W=a constant } \\
\left.\frac{d \tau}{d \theta}=\sqrt{\sqrt{(1+\nu) a}} \frac{e}{\sqrt{1-e^{2}}\left[2-\sqrt{1-e^{2}}\right.}\right]^{2} \frac{1}{\partial W} \\
\frac{d g}{d \tau}=\frac{2}{\sqrt{(1+\nu) a}} a \frac{\partial R}{\partial a}+\frac{1}{2-\sqrt{1-e^{2}}} \frac{d \theta}{d \tau} \\
-\frac{1}{\sqrt{(1+\nu) a}}\left[2-\sqrt{1-e^{2}}\right]^{2}+\frac{2}{2-\sqrt{1-e^{2}}}
\end{array}\right.
$$

$W$ has the expression :

$$
\begin{aligned}
W= & \frac{1}{2 a}\left[2-\sqrt{1-e^{2}}\right]^{2}+\sqrt{(1+\nu) a} \frac{1-e^{2}+2 \sqrt{1-e^{2}}}{3+e^{2}}+\nu \frac{1}{\pi} \int_{0}^{\pi} \frac{d \gamma}{\Delta} \\
=\frac{1}{2 a} & {\left[1+e^{2}+\frac{1}{2} e^{4}+\frac{1}{4} e^{6}+\frac{5}{32} e^{8}+\frac{7}{64} e^{10}+\cdots\right] } \\
& \quad+\sqrt{(1+\nu) a}\left[1-e^{2}+\frac{1}{4} e^{4}-\frac{1}{8} e^{6}+\frac{1}{6} \frac{1}{4} e^{8}-\frac{3}{12} e^{10}+\cdots\right]+R
\end{aligned}
$$

This equation contains as variables only $e$ and $\theta$; hence, since $e$ should never be negative, the dependence of the two variables on each other may be shown graphically by taking $e$ as the radius and $\theta$ as the angle in a system of polar coördinates. If we are given a pair of simultaneous values of $e$ and $\theta$, it is obvious that by their aid we can determine the constant value of $W$. Desiring to ascertain at what points on the axis the curve passes we make in (61) in succession $\theta=0^{\circ}$ and $\theta=180^{\circ}$ and we get two equations of the forms :

$$
\left\{\begin{array}{l}
D=M_{1} e+M_{2} e^{2}+M_{3} e^{3}+M_{4} e^{4}+M_{5} e^{5}+\cdots, \\
D=-M_{1} e+M_{2} e^{2}-M_{3} e^{3}+M_{4} e^{4}-M_{5} e^{5}+\cdots,
\end{array}\right.
$$

where $D$ may be regarded as the arbitrary constant and the $M$ are constants, being functions of $a$ and $\nu$. These equations are transcendental in $e$ and are such that the positive roots of the one are equivalent to the negative roots of the 
other. If each has a positive real root continuous with the value of $e$ which was used for the determination of the constant value of $W$, the variable $\theta$ generally moves through the whole range of real values. But, if the first equation has two positive real roots and the second none, $\theta$ will librate about the value $\theta=0^{\circ}$. But, if the second has two positive real roots and the first none, $\theta$ will librate about $\theta=180^{\circ}$. It will be seen that when $D=0$ we have the limit separating non-libration from libration.

$$
\mathrm{XI}_{i}^{\text {ins }}
$$

Case I.-Non-libration.-Here, as $\theta$ goes through the semicircumference, it can be employed as the independent variable. Then, in the first equation of $(60)$, we assign to $\theta$, in succession, a series of equidistant values covering the semicircumference. (Those used in our illustrative examples are 13 in number, viz, $\theta=0^{\circ}, \theta=15^{\circ}, \theta=30^{\circ}, \cdots, \theta=180^{\circ}$.) This procedure furnishes us with a like number of equations for determining the corresponding values of $e$. Solving these by the tentative process we have these values of $e$, and can apply to them the procedure of mechanical quadratures. Thus is obtained a general expression for $e$ as a periodic function of $\theta$ involving only cosines.

As the next step these special values of $e$ can be substituted in the right member of the second equation of $(60)$. To the special values thus obtained for $d \tau / d \theta$ can be applied mechanical quadratures, and the resulting periodic series, involving only cosines of integral multiples of $\theta$, can be integrated with respect to this variable. This integral may be put in the form :

$$
\theta_{0}(t+c)=\theta+\beta_{1} \sin \theta+\beta_{2} \sin 2 \theta+\beta_{3} \sin 3 \theta+\cdots
$$

Knowing $\theta_{0}$ we are now in possession of the period of the inequalities we are endeavoring to derive. The left member of this equation we shall designate as the time-argument, and, for brevity, denote it as $\zeta$. In the next place we assign to $\zeta$ a series of equidistant values going from $0^{\circ}$ to $180^{\circ}$, and, by a tentative process applied to (63), arrive at the corresponding values of $\theta$. These corresponding values of $\theta$ can be substituted for $\theta$ in the expression of $e$ as a periodic function of $\theta$, and thus we shall have the values of $e$ whieh correspond to the equidistant values of $\zeta$. We can now readily derive the similar values of the two quantities $e \cos \theta$ and $e \sin \theta$. To these we apply mechanical quadratures and thus obtain the periodic developments of these quantities in terms of $\zeta$.

As the last step in this work we can, through the last equation of (60), express $d g / d \tau$ as a function of $e$ and $\theta$, and, by the substitution of the special values of the latter variables, obtain the special values of $d g / d \tau$ which correspond to the equidistant values of $\zeta$. To these apply mechanical quadratures and the periodic series for $d g / d \tau$ is obtained. This being integrated we have the series for $g$, and the solution of the problem is completed. 
XII.

Case II.-Libration.-Here we are iut off from the use of $\theta$ as an independent variable on account of its not foing through the semicircumference. But this difficulty is surmounted by substituting fo, it another variable which does move continuously from $-\infty$ to $+\infty$. In order to ascertain, in the case of libration, the limiting values of $\theta$ we have to solve the simultaneous equations:

$$
W=a \text { constant }, \quad \frac{\partial W}{\partial e}=0,
$$

the unknowns being $e$ and $\theta$. That is to say, a value of $\theta$ must be found which will make the first equation have two equal roots for $e$. This can be done by a tentative process. If we assume $\theta$ too large, generally, we shall not be able to discover real values for $e$ from the first equation; but, if $\theta$ is taken too small, we get two values real but unequal for $e$. These two conditions must be brought as close as possible until we discover the point of passage from one to the other. In our illustrative example we escape the necessity of this tentative process by assuming as one of the two fundamental elements of the example not the $D$ of (62) but the amount of libration.

The amount of libration being thus either assumed or determined, let $\kappa$ denote the limiting value of $\sin \theta$; we then can put

$$
\sin \theta=\kappa \sin \psi
$$

and the motion of $\psi$ can be regarded as extending continuously from $-\infty$ to $+\infty$. Adopting the variable $\psi$ for replacing $\theta$, the second equation of $(60)$ takes the form :

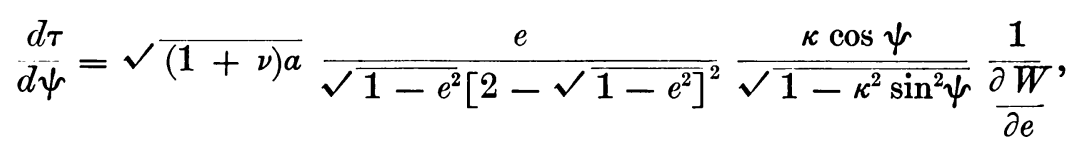

where the newly introduced radical must receive the $\operatorname{sign}$ of $\cos \theta$. We can now make $\psi$ play the same rôle as $\theta$ did in Case $I$, and there is need of no further explanations.

\section{XIII.}

We attend now to the integration of equations (60). The operation of Delaunay's lunar theory which is numbered 23* has great affinity with that here detailed, and the two may be compared. $\mathrm{He}$, it is true, has six variables to our four; but, in comparing, his $\gamma$ should be made to vanish and his $h$ then becomes indeterminate.

\footnotetext{
* Mémoires de l'Académie des Sciences, vol. XXVIII, p. 493.
} 
The periodic development of the reciprocal of the distance between two planets as a function of the time has been given by LEVERRIER to terms of the seventh order inclusive, and th $\sim e$ of the eighth order have afterwards been added by M. BouQUET.* We avc il ourselves of this development and adopt the mode of LEVERRIER for noting the coefficients except in the portion which is a function of $e$ alone. We put $A_{j}^{(i)}=(1 / j !) a^{j} d^{j} b_{\frac{1}{2}}^{(i)} / d a^{j}, j=0$ in the portion factored by $\cos 0 \theta, j=2$ in the portion factored by $\cos \theta, j=4$ in the portion factored by $\cos 2 \theta$ and so on ; only the numerical factors are written since the $A$ can easily be filled in as they always commence with $A_{0}^{(\Uparrow)}$, and the lower index always increases by a unit in each step to the right. With Leverrien we put $\chi$ for $\frac{1}{2} e$. This then is the development of $a^{\prime} / \Delta$, preserving only the terms involving the integral multiples of $\theta$ as arguments :

$$
\begin{aligned}
& \frac{a^{\prime}}{\Delta}=\frac{1}{2} A_{0}^{(0)}+\left[A_{1}^{(0)}+A_{2}^{(0)}\right] \chi^{2}+{ }_{1}^{3}\left[A_{3}^{(0)}+A_{4}^{(0)}\right] \chi^{4}+\frac{4.5}{1.2}\left[A_{5}^{(0)}+A_{6}^{(0)}\right] \chi^{6}+\frac{5.6 .7}{1.2 .3}\left[A_{7}^{(0)}+A_{8}^{(0)}\right] \chi^{8} \\
& +\left\{-[4+1] x+\left[14+\frac{5}{2}-6-3\right] x^{3}+\left[-\frac{5}{3}-\frac{53}{12}+\frac{34}{3}+5-16-10\right] x^{5}\right. \\
& \left.+\left[\frac{271}{36}-\frac{203}{144}+\frac{19}{4}-\frac{49}{8}+20+\frac{25}{2}-50-35\right] \chi^{7}\right\} \cos \theta \\
& +\left\{[22+7+1] \chi^{2}+\left[-\frac{596}{3}-\frac{212}{3}+8+16+4\right] \chi^{4}+\left[\frac{1300}{3}+\frac{743}{3}-49\right.\right. \\
& -112+2+45+15] \chi^{6}+\left[-\frac{8312}{45}-\frac{500212}{45}-\frac{280}{9}+\frac{1228}{5}\right. \\
& \left.+0-236-16+140+56] \chi^{8}\right\} \cos 2 \theta \\
& +\left\{-\left[134+\frac{93}{2}+10+1\right] \chi^{3}+\left[\frac{4053}{2}+\frac{6289}{8}+107-\frac{117}{2}-32-5\right] \chi^{5}\right. \\
& \left.+\left[-\frac{64177}{40}-\frac{384789}{80}-\frac{12123}{20}+\frac{6249}{8}+348-\frac{189}{2}-102-21\right] \chi^{7}\right\} \cos 3 \theta \\
& +\left\{\left[\frac{2570}{3}+\frac{932}{3}+80+13+1\right] \chi^{4}+\left[-\frac{275528}{15}-\frac{109972}{15}-1676+48+176\right.\right. \\
& +54+6] \chi^{6}+\left[\frac{6259444}{45}+\frac{592976}{9}+\frac{234338}{15}-\frac{44756}{15}-\frac{10036}{3}-\frac{1432}{3}\right. \\
& \left.+400+196+28] \chi^{8}\right\} \cos 4 \theta \\
& +\left\{-\left[\frac{33797}{6}+\frac{50345}{24}+\frac{1795}{3}+\frac{245}{2}+16+1\right] \chi^{5}+\left[\frac{5652235}{36}+\frac{9141589}{144}+\frac{210217}{12}\right.\right. \\
& \left.\left.+\frac{48985}{24}-\frac{2020}{3}-\frac{775}{2}-82-7\right] \chi^{7}\right\} \cos 5 \theta \\
& +\left\{\left[\frac{188616}{5}+\frac{71499}{5}+4357+1024+174+19+1\right] \chi^{6}+\left[-\frac{45378132}{35}\right.\right. \\
& \left.\left.-\frac{3695460}{7}-\frac{803616}{5}-\frac{150828}{5}+80+2156+720+116+8\right] \chi^{8}\right\} \cos 6 \theta
\end{aligned}
$$

* Annales de l'Observatoire de Paris, vols. I, XIX. 


$$
\begin{gathered}
+\left\{-\frac{46064791}{2520}+\frac{70738549}{720}+\frac{1880921}{60}+\frac{193921}{24}+\frac{4844}{3}+\frac{469}{2}-22-1\right\} x^{7} \cos 7 \theta \\
+\left\{\frac{552146674}{315}+\frac{213998824}{315}+\frac{2018552}{9}+\frac{926516}{15}+\frac{41380}{3}+\frac{7192}{3}\right. \\
+304+25+1\} x^{8} \cos 8 \theta .
\end{gathered}
$$

From this expression we must eliminate the variable $a$ by means of its value in terms of $e$ given by (57). We put

$$
p=\left[2-\sqrt{1-e^{2}}\right]^{-2}-1 .
$$

Let LeVERRIER's coefficient of $\cos j \theta$ be denoted thus :

$$
c_{0} A_{0}^{(2 j)}+c_{1} A_{1}^{(2 j)}+c_{2} A_{2}^{(2 j)}+\cdots=\sum c_{i} A_{i}^{(2 j)},
$$

where $c_{i}$ is a function of $e$. Denoting the similar coefficient, after the variable $a$ has been eliminated through (57) by $\sum f_{i} A_{i}^{(2 j)}$, we evidently have

$$
f_{i}=(1+p)^{i} c_{i}^{*}+\frac{i}{1}(1+p)^{i-1} p c_{i-1}+\frac{i(i-1)}{1.2}(1+p)^{i-2} p^{2} c_{i-2}+\cdots
$$

By means of this formula we obtain the following expression, in which $a$, the argument of the various quantities $A_{i}^{(2 j)}$, is the constant $a$ of (57):

$$
\begin{aligned}
& \frac{a^{\prime}}{\Delta}=\frac{1}{2}+[0-1+1] \chi^{2}+[0+0-8-9+3] \chi^{4}+[0+0+48+100+0-50+10] x^{6} \\
& +[0-4-160-600-224+720+240-245+35] x^{8} \\
& +\left\{-[4+1] \chi+\left[14+\frac{45}{2}+2-3\right] \chi^{3}+\left[-5^{-}-\frac{1325}{12}-\frac{218}{3}+65+32-10\right] \chi^{5}\right. \\
& \left.+\left[\frac{271}{36}+\frac{33829}{144}+\frac{6209}{12}-\frac{3025}{8}-796+\frac{105}{2}+190-35\right] \chi^{7}\right\} \cos \theta \\
& +\left\{[22+7+1] x^{2}+\left[-\frac{596}{3}-\frac{560}{3}-56+4+4\right] x^{4}+\left[\frac{1300}{3}+1557+\frac{3517}{3}\right.\right. \\
& +248-222-35+15] x^{6}+\left[-\frac{8312}{45}-\frac{740632}{45}-\frac{95032}{9}-\frac{19112}{5}\right. \\
& \left.+3680+2184-496-280+56] x^{8}\right\} \cos 2 \theta \\
& +\left\{-\left[134+\frac{93}{2}+10+1\right] \chi^{3}+\left[\frac{4053}{2}+\frac{12065}{8}+559+\frac{147}{2}-16-5\right] \chi^{5}\right. \\
& \left.+\left[-\frac{64177}{40}-\frac{1400349}{80}-\frac{248943}{20}-\frac{26439}{8}+612+\frac{971}{2}+18-21\right] \chi^{7}\right\} \cos 3 \theta \\
& +\left\{\left[\frac{2570}{3}+\frac{932}{3}+80+13+1\right] x^{4}+\left[-\frac{275528}{15}-\frac{60004}{5}-\frac{14404}{3}-1068-48\right.\right. \\
& +34+6] \chi^{6}+\left[\frac{6259444}{45}+\frac{1602224}{9}+\frac{1180994}{15}+\frac{630004}{15}\right. \\
& \left.\left.+\frac{11372}{3}-\frac{7912}{2}-800+28+28\right] \chi^{8}\right\} \cos 4 \theta
\end{aligned}
$$




$$
\begin{aligned}
& +\left\{-\left[\frac{33797}{6}+\frac{50345}{24}+\frac{1795}{3}+\frac{245}{2}+16+1\right] \chi^{5}+\left[\frac{5652235}{36}+\frac{13594381}{144}\right.\right. \\
& \left.\left.+\frac{469037}{12}+\frac{256585}{24}+\frac{4628}{3}-\frac{95}{2}-58-7\right] \chi^{7}\right\} \cos 5 \theta \\
& +\left\{\left[\frac{188616}{5}+\frac{71499}{5}+4357+1024+174+19+1\right] \chi^{6}+\left[-\frac{45378432}{35}\right.\right. \\
& \left.\left.-\frac{5152104}{7}-\frac{1549888}{5}-\frac{473688}{5}-19088-1704+240+88+8\right] \chi^{8}\right\} \cos 6 \theta \\
& +\left\{-\frac{46064791}{2520}+\frac{70738549}{720}+\frac{1880921}{60}+\frac{191863}{24}+\frac{4844}{3}+\frac{469}{2}+21+1\right\} \chi^{7} \cos 7 \theta \\
& +\left\{\frac{552146674}{315}+\frac{213998824}{315}+\frac{2018552}{9}+\frac{926516}{15}+\frac{41380}{3}+\frac{7192}{3}+304+25+1\right\} \chi^{8} \cos 8 \theta
\end{aligned}
$$

In forming the value of $d g / d \tau$ we need to know the derivative of the foregoing expression with respect to $a$. By noting the equation :

$$
a \frac{\partial A_{i}^{(2 j)}}{\partial a}=i A_{i}^{(2 j)}+(i+1) A_{i+1}^{(2 j)},
$$

and changing our mode of noting the coefficients so that the number first given is the coefficient of $A_{1}^{(2 j)}$ instead of $A_{0}^{(2 j)}$, we have:

$$
\begin{aligned}
& a \frac{\partial \frac{a^{\prime}}{\Delta}}{\partial a}=\frac{1}{2}+[-1+0+3] \chi^{2}+[0-16-51-24+15] \chi^{4}+[0+96+444+400-250 \\
& -240+70] \chi^{6}+[-4-328-2280-3296+2480+5760-35-1680+315] \chi^{8} \\
& +\left\{-[5+2] \chi+\left[\frac{73}{2}+49-3-12\right] \chi^{3}+\left[-\frac{1345}{12}-\frac{2197}{6}-23+388+110-60\right] \chi^{5}\right. \\
& \left.+\left[\frac{34913}{144}+\frac{108337}{72}+\frac{3343}{8}-\frac{9093}{2}-\frac{7435}{2}+1455+1085-280\right] \chi^{7}\right\} \cos \theta \\
& +\left\{[29+16+3] \chi^{2}+\left[-\frac{1156}{3}-\frac{1456}{3}-156+32+20\right] \chi^{4}+\left[\frac{5971}{3}\right.\right. \\
& \left.+\frac{16376}{3}+4261+104-1285-120+105\right] \chi^{6}+\left[-\frac{83216}{5}-\frac{270176}{5}\right. \\
& \left.\left.-\frac{647168}{15}-\frac{2848}{5}+29320+10128-5432-1792+504\right] \chi^{8}\right\} \cos 2 \theta \\
& +\left\{-\left[\frac{361}{2}+113+33+4\right] \chi^{3}+\left[\frac{28277}{8}+\frac{16537}{4}+\frac{3795}{2}+230-105-30\right] \chi^{5}\right. \\
& +\left[-\frac{1528703}{80}-\frac{2396121}{40}-\frac{1890243}{40}-\frac{21543}{2}+\frac{11975}{2}\right. \\
& \left.+3021-21-168] \chi^{7}\right\} \cos 3 \theta \\
& +\left\{\left[\frac{3502}{3}+\frac{2344}{3}+279+56+5\right] \chi^{4}+\left[-\frac{91108}{3}-\frac{504064}{15}-17608-4464\right.\right. \\
& -70+240+42] \chi^{6}+\left[\frac{14270564}{45}+\frac{23108204}{45}+\frac{1810998}{5}+\frac{2747456}{15}\right. \\
& \left.\left.+\frac{17300}{5}-20624-5404+448+252\right] \chi^{8}\right\} \cos 4 \theta
\end{aligned}
$$




$$
\begin{aligned}
& +\left\{-\left[\frac{185533}{24}+\frac{64705}{12}+\frac{4325}{2}+554+85+6\right] \chi^{5}+\left[\frac{36203321}{144}+\frac{19222825}{72}\right.\right. \\
& \left.\left.+\frac{1194659}{8}+\frac{293609}{6}+\frac{44855}{6}-633-455-56\right] \chi^{7}\right\} \cos 5 \theta \\
& +\left\{\left[52023+\frac{186568}{5}+16143+4792+965+120+7\right] x\right. \\
& +\left[-\frac{71138952}{35}-\frac{73219472}{35}-\frac{6070728}{5}-\frac{2276512}{5}-103960-8784\right. \\
& \left.+2296+768+72] \chi^{8}\right\} \cos 6 \theta \\
& -\left\{\frac{587299425}{5040}+\frac{93309601}{360}+\frac{4721157}{40}+\frac{230615}{6}+\frac{55475}{6}+1539+161+8\right\} \chi^{7} \cos 7 \theta \\
& +\left\{\quad \frac{766145498}{315}+\frac{569296288}{315}+\frac{12872308}{15}+\frac{4533664}{15}+\frac{242860}{3}+16208\right. \\
& +2303+208+9\} x^{8} \cos 8 \text {. }
\end{aligned}
$$

It is desirable to have the means of verifying these truncated developments of $a^{\prime} / \Delta$ derived from the work of Leverrier and M. Bouquet. In fact, by the application of the first of two following theorems, an error has been found in M. BouqueT's expression for (225); in the coefficient of $K_{3},-h$ should be substituted for $h$. The two theorems are the following:

The coefficient of $\cos j \theta$ in the periodic development of $a^{\prime} / \Delta$ is the same as that of $s^{j}$ in the expansion of the expression

$$
\sum_{i=0}^{i=\infty} A_{i}^{(2 j)}(-\chi)^{i}\left(s+\frac{1}{s}\right)^{i}\left[1-\chi\left(s+\frac{1}{s}\right)\right]\left(\frac{s-\omega}{1-\omega s}\right)^{2 j} \epsilon^{j \chi\left(s-\frac{1}{s}\right)}
$$

in a power series with reference to $s$.

The coefficient of $\cos j \theta$ in the same development after a has been replaced by $a\left[2-\sqrt{ } 1-e^{2}\right]^{2}$ is the same as that of $s^{j}$ in the expansion of the expression

$$
\sum_{i=0}^{i=\infty} A_{i}^{(2 j)}\left[p-\chi(1+p)\left(s+\frac{1}{s}\right)\right]^{i}\left[1-\chi\left(s+\frac{1}{s}\right)\right]\left(\frac{s-\omega}{1-\omega s}\right)^{2 j} \epsilon^{j \chi\left(s-\frac{1}{s}\right)}
$$

in a power series with reference to $s$.

In these expressions $\omega$ stands for $e /\left(1+\sqrt{1-e^{2}}\right)$.

\section{XIV.}

The two linear elements which determine all the coefficients in the periodic developments involved in this problem may be taken to be the constant $a$ of (57) and the constant $D$ of (62). It is proposed to elaborate two examples illustrating the subject in hand, one exhibiting non-libration, the other libration. In both we will assign to $a$ such a value as makes $\log a=9.8$. This value makes the period of revolution of the small planet nearly or exactly half that of 
Jupiter. Whether we are to have a case of non-libration or libration will then depend on the value assigned to the second constant $D$.

In the first place then we compute the values of such of the quantities $A_{i}^{(j)}$ as are needed in this investigation, correspon? nt to $\log a=9.8$, by procedures which it is unnecessary to detail. The results are contained in the following table :

\begin{tabular}{|c|c|c|c|c|c|}
\hline & \multicolumn{5}{|c|}{ Values of $\log A_{i}^{(j)}$ for $\log a=9.8$. } \\
\hline$S_{1}$ & $i=0$ & $i=1$ & $i=2$ & $i=\mathbf{3}$ & $i=4$ \\
\hline 0 & $0.354 \quad 4041 \quad 774$ & $9.845 \quad 4797897$ & $9.9350116 \quad 655$ & 9.9891230 & 0.1113716 \\
\hline 2 & $9.564 \quad 3962 \quad 993$ & 9.96583671 & 0.00274635 & 0.0077852 & 0.1212342 \\
\hline 4 & 9.0350709047 & 9.69498971 & 9.99260306 & 0.0988192 & 0.1641565 \\
\hline 6 & 8.5550516205 & 9.37416115 & 9.84274420 & 0.0995969 & 0.2313986 \\
\hline 8 & 8.096854986 & $9.0317132 \quad 5$ & $9.624 \quad 74408$ & 0.0055654 & 0.2395776 \\
\hline 10 & 7.65106345 & 8.67703269 & 9.36743852 & 9.8492650 & 0.1788047 \\
\hline 12 & 7.21329428 & 8.31444617 & 9.08468372 & 9.6495418 & 0.0628948 \\
\hline 14 & 6.7811495 & 7.94631868 & 8.78414088 & 9.4196593 & 9.9052677 \\
\hline 16 & 6.3531544 & 7.5740873 & 8.4704851 & 9.1671668 & 9.7158220 \\
\hline$j$ & $i=5$ & $i=6$ & $i=7$ & $i=8$ & $i=9$ \\
\hline 0 & 0.2512555 & 0.4085603 & 0.576748 & 0.75337 & 0.9370 \\
\hline 2 & 0.2574382 & 0.4127086 & 0.579698 & 0.75557 & 0.9385 \\
\hline 4 & $0.278 \quad 2372$ & 0.4260622 & 0.588970 & 0.76242 & 0.9439 \\
\hline 6 & 0.3294253 & 0.4532441 & 0.606143 & 0.77466 & 0.9568 \\
\hline 8 & 0.3851308 & 0.5033035 & 0.637049 & 0.79430 & 0.9771 \\
\hline 10 & 0.3989502 & 0.5525313 & 0.684255 & 0.82657 & 1.0045 \\
\hline 12 & 0.3604400 & 0.5703163 & 0.729206 & 0.87086 & 1.0290 \\
\hline 14 & 0.2723635 & 0.5456897 & $0.750 \quad 571$ & 0.90938 & 1.0455 \\
\hline 16 & $0.146 \quad 2268$ & $0.480 \quad 0088$ & 0.721959 & $0.935 \quad 78$ & 1.0457 \\
\hline
\end{tabular}

Substituting these values in (67) we get

$$
\begin{aligned}
& \frac{a^{\prime}}{\Delta}=1.1307697497+0.04010033 e^{2}-0.7367846 e^{4}+1.17661 e^{6}+0.6155 e^{8} \\
& +\left[-1.19571949+3.1113902 e^{2}-2.669146 e^{4}-1.90033 e^{6}\right] e \cos \theta \\
& +\left[1.70905245-9.8883917 e^{2}+30.18579 e^{4}-62.2057 e^{6}\right] e^{2} \cos 2 \theta \\
& +\left[-3.00445698+27.190861 e^{2}-117.01214 e^{4}\right] e^{3} \cos 3 \theta \\
& +\left[5.7966694-71.99282 e^{2}+369.2943 e^{4}\right] e^{4} \cos 4 \theta \\
& +\left[-11.800399+186.12652 e^{2}\right] e^{5} \cos 5 \theta \\
& +\left[24.86635-475.7506 \quad e^{2}\right] e^{6} \cos 6 \theta \\
& \text { - } \quad 52.40299 e^{7} \cos 7 \theta \\
& +\quad 118.0918 e^{8} \cos 8 \theta \text {. }
\end{aligned}
$$

\begin{tabular}{|c|c|c|}
\hline \\
\hline-0.00226 & 075432 & $e^{2}$ \\
\hline+0.59490 & 01358 & $e^{4}$ \\
\hline+0.09877 & 323 & $e^{6}$ \\
\hline+0.13623 & 71 & $e^{8}$ \\
\hline+0.06804 & & $e^{10}$ \\
\hline
\end{tabular}

We adopt the mass of Jupiter so that $\nu=1 / 1047.355$. Then, in the expression (61) of $W$, the portion which is independent of the interaction of Jupiter and the small planet, developed in powers of $e^{2}$, becomes :

If we omit from the expansion of $W$ its constant term, and call $D$ the constant of the thus modified $W$, as in (62), we have, as an integral of our problem, 


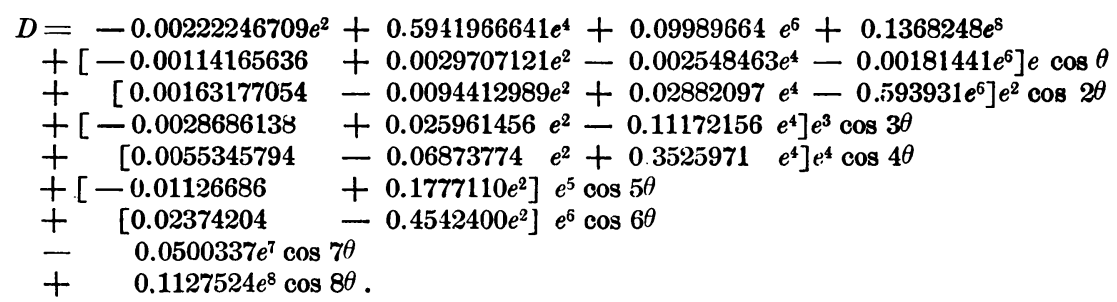

By making $\theta=0^{\circ}$ in the preceding equation, we get, as the correspondent of the first equation of (62), the following :

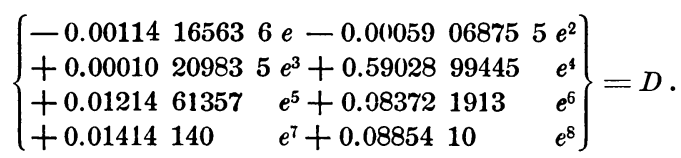

It will be seen by comparison of the coefficients of this equation that, unless $e$ is very small, it will not do to regard the equation as approximately a quadratic in $e$; for $e=0.1$ the term in $e^{4}$ is ten times more important than the term in $e^{2}$. The supposition that the mean motion of the small planet is nearly double that of Jupiter makes the coefficient of $e^{2}$ nearly vanish. In fact a very small change in the adopted value of $a$ would make this coefficient 0 .

What sort of a curve we shall have exhibiting graphically the connection between $e$ and $\theta$ will depend on the value assigned to $D$. To bring this out in a clear manner we compute the values of the left member of the preceding equation for each 0.01 in the value of $e$ between the limits \pm 0.3 , and thus have the following table :

\begin{tabular}{|c|c|c|c|c|c|c|c|}
\hline$e$ & $D$ & $e$ & $D$ & $e$ & $D$ & $e$ & $D$ \\
\hline-0.30 & +0.00510216 & -0.15 & +0.00045648 & +0.01 & -0.00001148 & +0.16 & +0.00019224 \\
\hline 0.29 & 448080 & 0.14 & 37472 & 0.02 & 2298 & 0.17 & 28623 \\
\hline 0.28 & 392021 & 0.13 & 30675 & 0.03 & 3430 & 0.18 & 40095 \\
\hline 0.27 & 341625 & 0.12 & 25066 & 0.04 & 4509 & 0.19 & 53896 \\
\hline 0.26 & 296473 & 0.11 & 20477 & 0.05 & 5485 & 0.20 & 70298 \\
\hline 0.25 & 256178 & 0.10 & 16715 & 0.06 & 6294 & 0.21 & 89590 \\
\hline 0.24 & 220365 & 0.09 & 13659 & 0.07 & 6857 & 0.22 & 112071 \\
\hline 0.23 & 188675 & 0.08 & 11166 & 0.08 & 7082 & 0.23 & 138067 \\
\hline 0.22 & 160764 & 0.07 & 9115 & 0.09 & 6861 & 0.24 & 167912 \\
\hline 0.21 & 136306 & 0.06 & 7400 & 0.10 & 6073 & 0.25 & 201960 \\
\hline 0.20 & 114987 & 0.05 & 5928 & 0.11 & 4582 & 0.26 & 240580 \\
\hline 0.19 & 96512 & 0.04 & 4622 & 0.12 & -0.00002236 & 0.27 & 284159 \\
\hline 0.18 & 80599 & 0.03 & 3420 & 0.13 & +0.00001129 & 0.28 & 333102 \\
\hline 0.17 & 66982 & 0.02 & 2269 & 0.14 & 5695 & 0.29 & 387834 \\
\hline-0.16 & +0.00055411 & -0.01 & +0.00001136 & +0.15 & +0.00011657 & +0.30 & † 0.00448802 \\
\hline
\end{tabular}

As $e$ ought always to be positive, in the first half of this table we may change the sign of $e$, provided we suppose that the corresponding value of $D$ is regarded as appertaining to the special value $180^{\circ}$ for $\theta$, while, in the remainder of the table, this value corresponds to $\theta=0^{\circ}$. 
From the course of the values of $D$ in the table we see there is one minimum $=-0.00007082$, which occurs for $e=0.08$ about; consequently, if $D$ is chosen greater than this the equation (71) will have two real roots for $e$. If $D$ is positive one of these roots will be negative ; changing the sign of the latter it will belong to the value $\theta=180^{\circ}$; the positive root will belong to $\theta=0^{\circ}$. Thus, in this case, the motion of $\theta$ is generally through the whole semicircumference, and hence is continuous from $-\infty$ to $+\infty$. But, if $D$ is negative, both roots will be positive, and thus belong to $\theta=0^{\circ}$. In this case, therefore, $\theta$ departs from $0^{\circ}$ and comes back to it without having reached $180^{\circ}$. This is called a libration; we see that $D=0$ marks the dividing point between continuous and libratory motion for $\theta$. The latter case also has the largest swing in the values of $e$, viz, from $e=0$ to about $e=0.127$. Generally, the larger $D$ is, the smaller will be the variation in $e$. Thus, if $D=+0.0045$, $e$ will vary from 0.29 to 0.30 . If there is libration $e$ cannot exceed 0.127 . These remarks, however, must be understood as applying only to the values holding for $\theta=0^{\circ}$ and $\theta=180^{\circ}$. Larger values for $e$ may obtain for values of $\theta$ lying between $0^{\circ}$ and $180^{\circ}$.

\section{XV.}

For our illustrative example, in the case of a continuous motion for $\theta$, we assign to $D$ the value +0.0001 in $(70)$. All the coefficients of the various periodic series will now have determinate numerical values. The preceding table shows that, for this assumption, the eccentricity will have, when $\theta=0^{\circ}$, the approximate value $e=0.1475$, and, when $\theta=180^{\circ}$, the approximate value $e=0.0745$. In this case these are the limiting values, as $e$ continuously diminishes while $\theta$ is passing from $0^{\circ}$ to $180^{\circ}$.

Attending now to the elaboration of our selected example, in (70) we give to $\theta$, in succession, the values $15^{\circ}, 30^{\circ}, 45^{\circ}, 60^{\circ}, 75^{\circ}, 90^{\circ}$ and get as the right member of $(70)$, [the value $\theta=0^{\circ}$ has already been considered in (70)],

\begin{tabular}{|c|c|c|c|c|}
\hline $15^{\circ}$ & $-0.0011027554 e$ & $-0.0008093046 e^{2}$ & $+0.000841072 e^{3}$ & $+0.58878755 e^{4}$ \\
\hline 30 & 9887034 & 14065774 & 2572712 & 58670872 \\
\hline 45 & 8072730 & 22224671 & 4129027 & $58866 \quad 208$ \\
\hline 60 & 5708282 & 30383568 & 4353970 & $59615 \quad 002$ \\
\hline 75 & -0.0002954824 & 36356296 & +0.002797293 & 60514036 \\
\hline 90 & $\begin{array}{r}0 \\
0\end{array}$ & -0.0038542466 & 0 & +0.60917254 \\
\hline$\theta$ & & & & \\
\hline $15^{\circ}$ & $+0.0129798 e^{5}$ & $+0.090487 e^{6}$ & $-0.021807 e^{7}$ & $+0.20531 e^{\delta}$ \\
\hline 30 & +00075503 & +0.124934 & -0.112143 & +0.32869 \\
\hline 45 & -0.0121927 & $\begin{array}{r}0.168634 \\
\end{array}$ & -0.083324 & -0.10302 \\
\hline 60 & -0.0328691 & +0.143597 & +0.174653 & -0.52039 \\
\hline 75 & -0.0299001 & +0.040568 & +0.298514 & +0.30818 \\
\hline 90 & 0 & -0.021404 & 0 & +1.11581 \\
\hline
\end{tabular}

The coefficients in the second quadrant for $\theta$ are the same as in the first, but in reverse order, except, that for the odd powers of $e$ the sign must be reversed. 
Making the right members of these 13 equations equal to +0.0001 , we solve them with reference to $e$ as the unknown, and substitute, in succession, the values thus obtained and the corresponding value of $\theta$ in the right member of the second equation of $(60)$. The results obtained are the following :

$\begin{array}{rcc}\theta & e & d \tau / d \theta \\ 0^{\circ} & 0.147462372 & 18.321384 \\ 15 & 0.147027366 & 18.443969 \\ 30 & 0.145692650 & 18.827993 \\ 45 & 0.143358947 & 19.521595 \\ 60 & 0.139828670 & 20.600475 \\ 75 & 0.134809503 & 22.197678 \\ 90 & 0.127912165 & 24.508178 \\ 105 & 0.118690066 & 27.615097 \\ 120 & 0.107094867 & 30.992715 \\ 135 & 0.094448152 & 32.866681 \\ 150 & 0.083602031 & 31.947061 \\ 165 & 0.076797488 & 29.983003 \\ 180 & 0.074547767 & 29.112462\end{array}$

The mean of the numbers in the third column, attributing half weight to the first and last, is 25.101781; and this is the number of revolutions of Jupiter in the period of the inequalities we are investigating. If the sidereal revolution of Jupiter is put at 11.861980 Julian years, the latter period is 297.75681 such years.

From the special values given in this table we can derive the two periodic series representing them. Integrating the latter, and, for brevity, putting $\zeta$ for $\theta_{0}(t+c)$, we get the following expressions :

$$
e=\left\{\begin{array}{ccc}
0.11918 & 891 & \\
+0 .(13553 & 171 \cos & \theta \\
-0.00857 & 010 \cos & 2 \theta \\
+0.00123 & 337 \cos 3 \theta \\
+0.00027 & 721 \cos & 4 \theta \\
-000029 & 767 \cos & 5 \theta \\
+0.00012 & 029 \cos & 6 \theta \\
-0.00001 & 828 \cos & 7 \theta \\
-0.00000 & 783 \cos & 8 \theta \\
+0.00000 & 780 \cos 9 \theta \\
-0.00000 & 374 \cos 10 \theta \\
+0.00000 & 037 \cos 11 \theta \\
+0.00000 & 033 \cos 12 \theta
\end{array}\right), \quad \zeta=\theta+\left\{\begin{array}{rrr}
-6017340 \sin & \theta \\
- & 1643.74 \sin & 2 \theta \\
+ & 4612.77 \sin & 3 \theta \\
- & 2132.15 \sin & 4 \theta \\
+ & 544.20 \sin & 5 \theta \\
+ & 6.13 \sin 6 \theta \\
- & 87.51 \sin 7 \theta \\
+ & 52.96 \sin 8 \\
- & 16.90 \sin 9 \theta \\
- & 0.02 \sin 10 \theta \\
+ & 3.89 \sin 11 \theta \\
- & 1.98 \sin 12 \theta
\end{array}\right\}
$$

The first of these is simply a transformation of the equation $W=D$ by which $e$ is expressed in terms of $\theta$. From the second, by attributing to $\zeta$ in succession the 13 values $0^{\circ}, 15^{\circ}, 30^{\circ}, \ldots, 180^{\circ}$, using a tentative process, we can get the corresponding values of $\theta$. Thence by substitution in former results, the corresponding values of the four quantities $e, e \cos \theta, e \sin \theta$ and $a \tau / d \theta$ can be obtained. The results follow, the first column containing the argument : 


\begin{tabular}{|c|c|c|c|c|c|}
\hline & $\theta$ & $e$ & $e \cos \theta$ & $e \sin \theta$ & $d \tau / d \theta$ \\
\hline $0^{\circ}$ & $0^{\circ} 0^{\prime} \quad 0^{\prime \prime} .00$ & $0.14746 \quad 237$ & +0.14746237 & 0.00000000 & 18.321384 \\
\hline 15 & 202758.05 & 0.14664872 & 0.13739211 & +0.05127624 & 18.551258 \\
\hline 30 & 402523.50 & 0.14418648 & 0.10976569 & 0.09349457 & 19.274084 \\
\hline 45 & $\begin{array}{lll}59 & 23 & 15.52\end{array}$ & 0.13999969 & $0.07129 \quad 163$ & 0.12048825 & 20.547277 \\
\hline 60 & $76 \quad 5725.45$ & 0.13402600 & +0.03024713 & 0.13056830 & 22.453723 \\
\hline 75 & 925150.00 & 0.12634244 & -0.00631251 & 0.12618464 & 25.044275 \\
\hline 90 & $107 \quad 342.77$ & 0.11722678 & 0.03439485 & $0.11206 \quad 745$ & 28.087856 \\
\hline 105 & $11948 \quad 15.87$ & 0.10725784 & $0.05331 \quad 151$ & 0.09307055 & 30.953203 \\
\hline 120 & 1313527.90 & 0.09727656 & 0.06457314 & 0.07275327 & 32.686384 \\
\hline 135 & $143 \quad 3 \quad 46.20$ & $0.08820 \quad 764$ & 0.07050393 & 0.05300740 & 32.666482 \\
\hline 150 & $154 \quad 48 \quad 43.11$ & 0.08092658 & $0.07323 \quad 176$ & 0.03444156 & 31.300251 \\
\hline 165 & $\begin{array}{lll}167 & 9 & 46.91\end{array}$ & 0.07619338 & 0.07428902 & +0.01692847 & 29.759410 \\
\hline 180 & $\begin{array}{lll}180 & 0 & 0.00\end{array}$ & 0.07454777 & -0.07454777 & 0.00000000 & 29.112462 \\
\hline
\end{tabular}

From the numbers in the fourth and fifth columns are derived the series:

$$
e \cos \theta=\left\{\begin{array}{llr}
+0.00071 & 143 \\
+0.10563 & 221 \cos & \zeta \\
+0.03542 & 782 \cos & 2 \zeta \\
+0.00539 & 467 \cos & 3 \zeta \\
+0.00031 & 839 \cos & 4 \zeta \\
-0.00002 & 420 \cos & 5 \zeta \\
-0.00000 & 130 \cos & 6 \zeta \\
+0.00000 & 245 \cos & 7 \zeta \\
+0.00000 & 111 \cos & 8 \zeta \\
+0.00000 & 026 \cos & 9 \zeta \\
-0.00000 & 044 \cos 10 \zeta \\
-0.00000 & 032 \cos 11 \zeta \\
+0.00000 & 030 \cos 12 \zeta
\end{array}\right\},
$$$$
e \sin \theta=\left\{\begin{array}{rrrr}
+0.11737 & 247 \sin & \zeta \\
+0.03373 & 708 \sin & 2 \zeta \\
+0.00528 & 997 \sin & 3 \zeta \\
+0.00035 & 675 \sin & 4 \zeta \\
-0.00001 & 633 \sin & 5 \zeta \\
-0.00000 & 316 \sin & 6 \zeta \\
-0.00000 & 095 \sin & 7 \zeta \\
-0.00000 & 062 \sin & 8 \zeta \\
+0.00000 & 041 \sin & 9 \zeta \\
+0.00000 & 018 \sin 10 \zeta \\
-0.00000 & 004 \sin 11 \zeta
\end{array}\right\} .
$$

These forms for the integrals of our problem are to be preferred since they can also be used for the case of libration.

\section{XVI.}

To complete the solution the periodic series giving the position of the perihelion must be derived. Using logarithms instead of the actual coefficients, the first term of the right member of the third equation of (60) has the expression :

$$
\begin{aligned}
\frac{2}{v^{\prime}(1+v) a} a \frac{\partial R}{\partial a} & =[6.9251786]+[7.126034] e^{2}-[8.00771] e^{4}+[7.9475] e^{6}+[8.7115] e^{8} \\
& +\left\{-[7.9015032]+[8.284709] e^{2}+[7.66255] e^{4}-[8.9051] e^{6}\right\} e \cos \theta \\
& +\left\{[8.3084076]-[9.068286] e^{2}+[9.55239] e^{4}-[9.7559] e^{6}\right\} e^{2} \cos 2 \theta \\
& +\left\{-[8.707357]+[9.66685] e^{2}-[0.2895] e^{4}\right\} e^{3} \cos 3 \theta \\
& +\left\{[9.104403]-[0.20025] e^{2}+[0.9097] e^{4}\right\} e^{4} \cos 4 \theta \\
& +\left\{-[9.50120]+[0.7008] e^{2}\right\} e^{5} \cos 5 \theta \\
& +\left\{\quad[9.89773]+[1.1811] e^{2}\right\} e^{6} \cos 6 \theta \\
& -[0.2839] e^{7} \cos 7 \theta \\
& +\quad[0.6902] e^{8} \cos 8 \theta .
\end{aligned}
$$

The remaining terms of this expression for $d g / d \tau$ can readily be derived from the values of $e$ and $d \tau / d \theta$ correspondent to the argument $\zeta$ which have just been given. Calling these the second part of $d g / d \tau$, we have the following results : 


First Part
+0.000049796
0.000059848
0.000121787
0.000237477
0.000391638
0.000595969
0.000843915
0.001099821
0.001319503
0.001465221
0.001535401
0.001562180
+0.001569151

\begin{tabular}{rr}
\multicolumn{1}{c}{ Second Part } \\
-0.00579 & 1193 \\
0.00572 & 9341 \\
0.00554 & 3667 \\
0.00509 & 7143 \\
0.00420 & 4082 \\
0.00270 & 5347 \\
-0.00024 & 2584 \\
+0.00326 & 3944 \\
0.00773 & 7116 \\
001284 & 0064 \\
0.01789 & 1412 \\
0.02178 & 6499 \\
+0.02328 & 0093
\end{tabular}

$d g / d \tau$
-0.005741397
0.005669493
0.005421880
0.004859666
0.003812444
-0.002109378
+0.000601331
0.004363765
0.009056619
0.014305285
0.019426813
0.023348679
+0.024849244

The quantities in the last column furnish the periodic series for $d g / d \tau$. The absolute term shows that the mean motion of the perihelion of the minor planet is 0.004900079 times the mean motion of Jupiter. The integration of this series gives the expression for $g$. These two expressions follow ; $(g)$ is the arbitrary constant added to complete the integral, and, in the second term, the unit of $t$ is a Julian year.

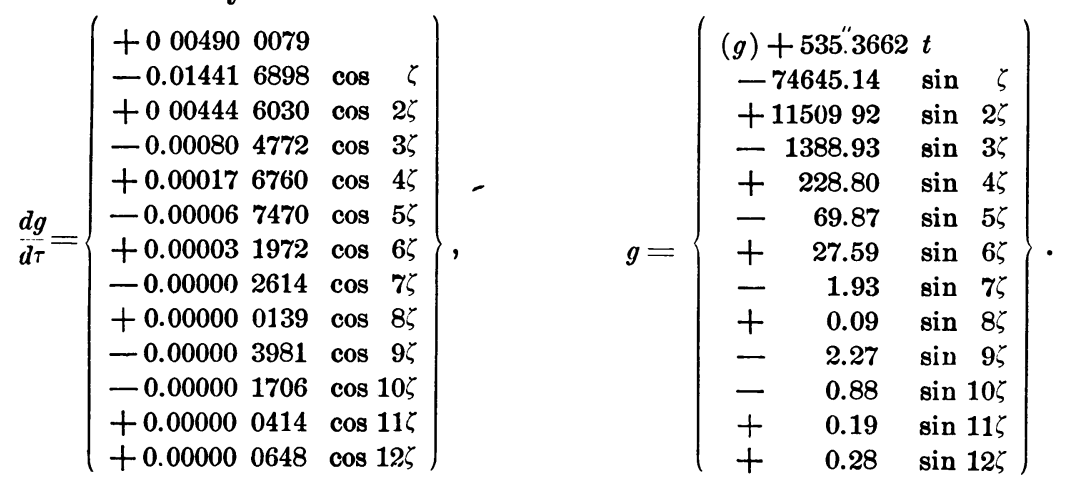

It is of interest to know the mean motion of the small planet which is not obvious at the beginning of the solution. We have the equation :

$$
\frac{d(l+g)}{d \tau}=\frac{d \theta}{d \tau}-\frac{d g}{d \tau}+2 .
$$

Substituting in the right member the mean motions of $\theta$ and $g$, its value is found to be 2.034937731 ; then, if for Jupiter we have $\mu^{\prime}=299$.' $^{\prime} 12838$, for the small planet $\mu=608$." 70762 .

\section{XVII.}

Illustration in the Case of Libration.-In the example we have chosen to illustrate the theory, libration, when it exists, is always about the value $\theta=0^{\circ}$. In addition to the value $\log a=9.8$ let us assume that the $D$ of (70) 
is to be so chosen that the half-swing of $\theta$ may be $50^{\circ}$. Making, therefore, $\theta=50^{\circ}$ in $(70)$, we get the first of the following equations in $e$, and the second by taking the derivative of the first with respect to $e$ :

$$
\left\{\begin{array}{ccc}
-0.00073 & 38426 & e \\
-0.00250 & 58226 & e^{2} \\
+0.00439 & 3829 & e^{3} \\
+0.58063 & 532 & e^{4} \\
-0.02026 & 791 & e^{5} \\
+0.17135 & 53 & e^{6} \\
-0.01446 & 68 & e^{7} \\
-0.324947 & e^{8}
\end{array}\right\}=D
$$$$
\left\{\begin{array}{lll}
-0.00073 & 38426 \\
-0.00501 & 16452 & e \\
+0.01318 & 1487 & e^{2} \\
+2.36254 & 128 & e^{3} \\
-0.10133 & 955 & e^{4} \\
+1.02813 & 18 & e^{5} \\
-0.10126 & 76 & e^{6} \\
-2.59957 & 6 & e^{7}
\end{array}\right\}=0 .
$$

Both of these equations should be satisfied when $\theta$ is at the limit of its swing, viz., when $\theta= \pm 50^{\circ}$. The root of the second equation which is applicable to our purpose is $e+0.07606124$, and this value substituted in the first gives $D=-0.0004863102$, which is the value of $D$ which brings about a libration of $50^{\circ}$.

From the equation $\sin \theta=\sin 50^{\circ} \sin \psi$ we obtain the following corresponding values :

\begin{tabular}{rrrr}
$\psi$ & & \multicolumn{1}{r}{$\theta$} & \\
$\circ$ & $\circ$ & \multicolumn{1}{c}{ " } \\
0 & 0 & 0 & 0.00 \\
15 & 11 & 26 & 8.27 \\
30 & 22 & 31 & 15.64 \\
45 & 32 & 47 & 51.90 \\
60 & 44 & 33 & 38.75 \\
75 & 47 & 43 & 35.34 \\
90 & 50 & 0 & 0.00
\end{tabular}

By means of these values we determine the form of (70) corresponding to the seven values of $\psi$. The coefficients are given in the following table (the small figures at the top of the columns denote the order of the final decimal):

\begin{tabular}{|c|c|c|c|c|c|c|c|c|}
\hline$\psi$ & $e$ & $e^{2}$ & $e^{3}$ & $e^{4}$ & $e^{5}$ & $e^{6}$ & $e^{7}$ & $e^{8}$ \\
\hline $0^{\circ}$ & $-11416564^{10}$ & $590688^{9}$ & $+10210^{8}$ & $+59028994^{8}$ & $+121461^{7}$ & $+83722^{6}$ & +1414 & $5+8854^{5}$ \\
\hline 15 & 11189923 & 718977 & 54217 & 58936012 & 128401 & 87139 & -640 & 15961 \\
\hline 30 & 10545925 & 1069471 & 164931 & 58751743 & 118852 & 103537 & $6636^{\circ}$ & 30349 \\
\hline 45 & 9596627 & 1548255 & 291587 & 58665083 & +48732 & 134345 & 12360 & 30000 \\
\hline 60 & 8542473 & 2027039 & 385516 & 58769012 & -67135 & 161748 & 11285 & +4731 \\
\hline 75 & 7679586 & 2377533 & 429468 & 58965923 & 166322 & 171341 & -4928 & -22703 \\
\hline & - 7338426 & -2505823 & +439383 & +59063532 & -202679 & +171355 & +1447 & -32495 \\
\hline
\end{tabular}

The expressions in this table constitute the left members of 7 equations of the 8 th degree ; they must be equated to the same quantity $D=-0.00004863102$. The two smallest real roots of each should be derived (they are those suited to our purpas). The connection of these roots with the variable $\psi$ is settled in following way: the larger of the two roots is made to correspond to the value of $\psi$ standing as the argument in the table, while the smaller is assigned to the value $180^{\circ}-\psi$; the two roots being equal for $\psi=90^{\circ}$, the common value is 
assigned to that value of $\psi$. This arrangement is made in order that $\psi$ and $\tau$ may augment together. These values of $e$ together with the corresponding values of $\theta$ are, in succession, substituted in (65); thus we have the values of $d \tau / d \psi$ corresponding to equidistant values of $\psi$. These resu... contained in the following table:

$\begin{array}{rccc}\psi & e & d \tau / d \psi \\ 0^{\circ} & 0.10846 & 187 & 37.23986 \\ 15 & 0.10765 & 795 & 37.70988 \\ 30 & 0.10518 & 818 & 39.14539 \\ 45 & 0.10088 & 191 & 41.57020 \\ 60 & 0.09452 & 189 & 44.76280 \\ 75 & 0.08605 & 349 & 47.65995 \\ 90 & 0.07606 & 124 & 48.00164 \\ 105 & 0.06601 & 592 & 44.40759 \\ 120 & 0.05744 & 757 & 38.68685 \\ 135 & 0.05102 & 653 & 33.34281 \\ 150 & 0.04671 & 683 & 29.47279 \\ 165 & 0.04426 & 767 & 27.22320 \\ 180 & 0.04347566 & 26.49213\end{array}$

It should be noted that, in the computation of the third column, the factor $\cos \psi /(\partial W / \partial e)$ takes on the indeterminate form $0 / 0$; employing the usual method of treating vanishing fractions, this factor equals $-1 /\left[\left(\partial^{2} W / \partial e^{2}\right)(d e / d \psi)\right]$. If here we should use the equation $W=D$ to determine $d e / d \psi$, the result would again be indeterminate. But this difficulty is avoided by employing the value of $e$ as a periodic function of $\psi$ given by the quantities of the second column. Thus if

then

$$
e=a_{0}+a_{1} \cos \psi+a_{2} \cos 2 \psi+a_{3} \cos 3 \psi+\cdots,
$$

$$
\frac{d e}{d \psi}=-a_{1} \sin \psi-2 a_{2} \sin 2 \psi-3 a_{3} \sin 3 \psi-\cdots
$$

and, for the special value $\psi=90^{\circ}$, this becomes :

$$
\frac{d e}{d \psi}=-a_{1}+3 a_{3}-5 a_{5}+7 a_{7}-\cdots
$$

For this special value of $\psi$ it is found that

$$
e \frac{\partial^{2} W}{\partial e^{2}}=+0.0028892836, \quad \frac{d e}{d \psi}=-0.03939373 .
$$

The mean of the numbers in the last column of the table, allowing half weight to the first and last, is 38.65409 , which is the number of revolutions of Jupiter contained in the period of libration; thus this period is 458.5144 Julian years. From the special values of $e$ and $d \tau / d \psi$ given in the table we derive the periodic series representing them. The latter can be integrated, and, as before, we put $\zeta$ for $\theta_{0}(t+c)$. Thus we get the following expressions : 


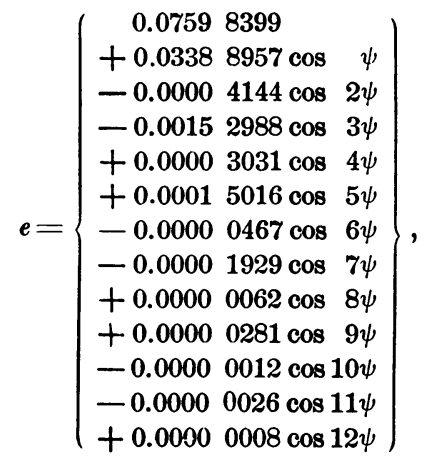$$
\zeta=\psi+\left\{\begin{array}{rrr}
+29862.37 \sin & \psi \\
-20922.46 \sin & 2 \psi \\
- & 416.31 \sin & 3 \psi \\
+ & 1650.00 \sin & 4 \psi \\
+ & 13.01 \sin & 5 \psi \\
- & 193.31 \sin 6 \psi \\
- & 0.37 \sin & 7 \psi \\
+ & 27.39 \sin & 8 \psi \\
+ & 0.01 \sin 9 \psi \\
- & 4.65 \sin 10 \psi \\
- & 0.02 \sin 11 \psi \\
+ & 0.81 \sin 12 \psi
\end{array}\right\} .
$$

The first of these is simply a transformation of the equation $W=D$, by which $e$ is expressed in terms of the auxiliary variable $\psi$.

Attributing to $\zeta$, in succession, the 13 values $0^{\circ}, 15^{\circ}, 30^{\circ}, \ldots, 180^{\circ}$, by a tentative process we can get the corresponding values of $\psi$, as also, by substitution those of $e, e \cos \theta$ and $e \sin \theta$. These results follow :

\begin{tabular}{|c|c|c|c|c|}
\hline$\zeta$ & $\psi$ & $e$ & $e \cos \theta$ & $e \sin \theta$ \\
\hline $0^{\circ}$ & $\begin{array}{llll}0 & 0 & 0.00\end{array}$ & 0.10846187 & +0.10846187 & 0.00000000 \\
\hline 15 & $\begin{array}{lll}15 & 30 & 0.64\end{array}$ & 0.10680283 & 0.10454084 & +0.02186455 \\
\hline 30 & 303558.93 & 0.10224969 & 0.09415541 & $0.0398 \quad 7176$ \\
\hline 45 & $\begin{array}{lll}44 & 58 & 46.75\end{array}$ & 0.09569588 & 0.08045265 & 0.05181767 \\
\hline 60 & $\begin{array}{lll}58 & 28 & 33.58\end{array}$ & 0.08797453 & 0.06662875 & 0.05744671 \\
\hline 75 & $\begin{array}{lll}71 & 8 & 20.78\end{array}$ & 0.07971930 & 0.05491390 & 0.05778952 \\
\hline 90 & $\begin{array}{lll}83 & 15 & 23.39\end{array}$ & 0.07144655 & 0.04637246 & 0.05435260 \\
\hline 105 & $\begin{array}{lll}95 & 20 & 46.24\end{array}$ & 0.06362489 & 0.04114864 & 0.04852749 \\
\hline 120 & 108715.59 & 0.05669409 & 0.03886515 & 0.04127614 \\
\hline 135 & $122 \quad 2527.98$ & 0.05107994 & 0.03896451 & 0.03302919 \\
\hline 150 & $\begin{array}{lll}139 & 5 & 9.66\end{array}$ & 0.04685171 & 0.04052863 & 0.02350559 \\
\hline 165 & 15831 & 0.04432198 & 0.04254216 & +0.01243393 \\
\hline 180 & 1800 & 0.04347566 & +0.04347566 & 0.00000000 \\
\hline
\end{tabular}

From the data of the fourth and fifth columns result the periodic series:

$$
e \cos \theta=\left\{\begin{array}{lllr}
+0.0604 & 2349 & \\
+0.0309 & 3374 & \cos & \zeta \\
+0.0147 & 2940 & \cos & 2 \zeta \\
+0.0015 & 8319 & \cos & 3 \zeta \\
+0.0007 & 3464 & \cos & 4 \zeta \\
-0.0000 & 1013 & \cos & 5 \zeta \\
+0.0000 & 6769 & \cos & 6 \zeta \\
-0.0000 & 1229 & \cos & 7 \zeta \\
+0.0000 & 1611 & \cos & 8 \zeta \\
-0.0000 & 0669 & \cos & 9 \zeta \\
+0.0000 & 0106 & \cos 10 \zeta \\
+0.0000 & 0528 & \cos 11 \zeta \\
-0.0000 & 0363 & \cos 12 \zeta
\end{array}\right\},
$$$$
e \sin \theta=\left\{\begin{array}{rrrr}
+0.0571 & 8416 & \text { sin } & \zeta \\
+0.0093 & 8541 & \text { sin } & 2 \zeta \\
+0.0030 & 1596 & \sin & 3 \zeta \\
+0.0000 & 5256 & \sin & 4 \zeta \\
+0.0001 & 9928 & \sin & 5 \zeta \\
-0.0000 & 1597 & \sin & 6 \zeta \\
+0.0000 & 1768 \text { sin } & 7 \zeta \\
-0.0000 & 0390 & \sin & 8 \zeta \\
+0.0000 & 0771 & \sin & 9 \zeta \\
-0.0000 & 0714 & \sin 10 \zeta \\
+0.0000 & 0492 & \sin 11 \zeta
\end{array}\right\} .
$$ 


\section{XVIII.}

In computing, for this case, the values of $d g / d \tau$ by the third equation of (60) we make a like division into two parts as in the former case. Substituting the values of $e$ and $\theta$ which correspond to the values $0^{\circ}, 15^{\circ}, 30^{\circ}, \ldots$, $180^{\circ}$ of $\zeta$ we get the following special values:

\begin{tabular}{|c|c|c|c|c|}
\hline$\zeta$ & First Part & Second Part & $d g / d \tau$ & \\
\hline $0^{\circ}$ & +0.00019583 & -0.05008981 & -0.04989 & 398 \\
\hline 15 & $20 \quad 725$ & 4843252 & 4822 & 527 \\
\hline 30 & 24656 & 4385892 & 4361 & 236 \\
\hline 45 & 30013 & 3722995 & 3692 & 982 \\
\hline 60 & 36434 & $2938 \quad 834$ & 2902 & 40 \\
\hline 75 & 42685 & $2098 \quad 623$ & 2055 & \\
\hline 90 & 47979 & 1247567 & 1199 & 588 \\
\hline 105 & 51817 & -0.00410869 & -0.00359 & 05 \\
\hline 120 & 54013 & +0.00397237 & +0.00451 & 25 \\
\hline 135 & 54781 & $1211 \quad 642$ & 1266 & 423 \\
\hline 150 & $54 \quad 497$ & 1991948 & 2046 & 44 \\
\hline 165 & 53746 & 2629903 & 2683 & 64 \\
\hline 180 & +0.00053360 & +0.02891583 & +0.02944 & 94 \\
\hline
\end{tabular}

From the quantities in the last column we obtain the periodic series for $d g / d \tau$, and thence by integration the expression for $g$; these results follow :

$$
\frac{d g}{d \tau}=\left\{\begin{array}{rrrr}
-0.0116 & 3174 & & \\
-0.0373 & 2443 & \cos & \zeta \\
+0.0007 & 9929 & \cos & 2 \zeta \\
-0.0020 & 0735 & \cos & 3 \zeta \\
+0.0005 & 0972 & \cos & 4 \zeta \\
-0.0002 & 9143 & \cos & 5 \zeta \\
+0.0000 & 8500 & \cos & 6 \zeta \\
-0.0000 & 4117 & \cos & 7 \zeta \\
+0.0000 & 1080 & \cos & 8 \zeta \\
-0.0000 & 0438 & \cos & 9 \zeta \\
+0.0000 & 0251 & \cos 10 \zeta \\
-0.0000 & 0293 & \cos 11 \zeta \\
+0.0000 & 0214 & \cos 12 \zeta
\end{array}\right\}, \quad g=\left\{\begin{array}{rrrr}
(g)-1270.844 t & \\
-297586.8 & \sin & \zeta \\
+ & 3186.4 & \sin & 2 \zeta \\
- & 5334.9 & \sin & 3 \zeta \\
+ & 1016.0 & \sin & 4 \zeta \\
- & 464.7 & \sin & 5 \zeta \\
+ & 113.0 & \sin & 6 \zeta \\
- & 46.9 & \sin & 7 \zeta \\
+ & 10.8 & \sin 8 \zeta \\
- & 3.9 & \sin 9 \zeta \\
+ & 2.0 & \sin 10 \zeta \\
- & 2.1 & \sin 11 \zeta \\
+ & 1.4 & \sin 12 \zeta
\end{array}\right)
$$

The unit of $t$ in the second expression is a Julian year.

By using the same formula as in the former case we find that the mean $\mu$ of the small planet in this case has the value $609^{\prime \prime} .47474$.

\section{XIX.}

In attempting to apply the preceding method to the case where $D=0$, we should find that $d g / d \tau$ became infinite at the point where $\theta=0^{\circ}$ or $\theta=180^{\circ}$, and, when $D$ is quite small, we should have to deal with inconveniently large numbers. This difficulty is surmounted by computing the differentials of the two quantities $e \cos g$ and $e \sin g$ in place of that of $g$. 